\title{
Double Diels-Alder Reactions of Linear Conjugated Tetraenes
}

\author{
Craig I. Turner, ${ }^{\dagger}$ Michael N. Paddon-Row, ${ }^{*}{ }^{*}$ Anthony C. Willis ${ }^{\dagger}$ and Michael S. Sherburn*, \\ Research School of Chemistry, Australian National University, Canberra, ACT 0200, Australia, and \\ School of Chemistry, The University of New South Wales, Sydney, NSW 2052, Australia. \\ E-mail: m.paddonrow@unsw.edu.au; sherburn@rsc.anu.edu.au
}

\section{Contents}

Computational Section - Cartesian coordinates of B3LYP/6-31+G(d) optimized TS geometries

Experimental Section - Synthetic details and compound characterization for compounds $1,3,4,5,6,7,8$ and 9

References S19

${ }^{1} \mathrm{H}$ and ${ }^{13} \mathrm{C}$ NMR spectra of compounds $1,3,4,5,6,7,8$ and 9
Page

S2-S12

S13-S18

S20-S35

\footnotetext{
$\dagger$ Australian National University

$\ddagger$ University of New South Wales
} 


\section{Computational Section}

\section{Internal-TS (Figure 2)}

MNP561 24-Apr-2004

B3LYP/6-31G(d) fully optimised TS + freq

maleic anhydride $+1,8$-dimethyloctatetraene internal attack ENDO TS

$\begin{array}{llcc}\text { C1 } & 1.4536932317 & -0.96656231 & 0.3540004282 \\ \text { C2 } & 0.6908363606 & -0.697871677 & 1.5155613617 \\ \text { C3 } & -0.6908363604 & -0.6978716773 & 1.5155613617 \\ \text { C4 } & -1.4536932314 & -0.9665623105 & 0.3540004282 \\ \text { C5 } & -0.7059291565 & 0.5800151326 & -0.9745823246 \\ \text { C6 } & 0.7059291563 & 0.5800151328 & -0.9745823246 \\ \text { C7 } & -1.1416423118 & 1.772156784 & -0.2107663615 \\ \text { C8 } & 1.1416423112 & 1.7721567844 & -0.2107663615 \\ \text { O9 } & 2.2375410774 & 2.230874578 & -0.0249417317 \\ \text { O10 } & -0.000000004 & 2.3607993297 & 0.3445231763 \\ \text { O11 } & -2.2375410781 & 2.2308745773 & -0.0249417317 \\ \text { H12 } & 1.0489484846 & -1.6848049327 & -0.3558480949 \\ \text { H13 } & 1.2119832224 & -0.3139395975 & 2.3902783052 \\ \text { H14 } & -1.2119832223 & -0.3139395979 & 2.3902783052 \\ \text { H15 } & -1.048948484 & -1.684804933 & -0.3558480949 \\ \text { H16 } & -1.3277750213 & 0.2188058216 & -1.782883597 \\ \text { H17 } & 1.3277750213 & 0.2188058221 & -1.782883597 \\ \text { C18 } & -2.911202581 & -0.8680718734 & 0.3647955927 \\ \text { C19 } & -3.7116740623 & -1.5227975979 & -0.4927315722 \\ \text { H20 } & -3.351582844 & -0.2069707951 & 1.1098218789 \\ \text { H21 } & -3.2559713415 & -2.1746274144 & -1.241335412 \\ \text { C22 } & 2.9112025813 & -0.8680718725 & 0.3647955927 \\ \text { C23 } & 3.7116740628 & -1.5227975967 & -0.4927315722 \\ \text { H24 } & 3.351582844 & -0.2069707941 & 1.1098218789 \\ \text { H25 } & 3.2559713422 & -2.1746274133 & -1.241335412 \\ \text { C26 } & -5.2071831632 & -1.439875399 & -0.5103648765 \\ \text { H27 } & -5.5827745219 & -0.7676234803 & 0.2672792122 \\ \text { H28 } & -5.5692481755 & -1.0740610187 & -1.4808928419 \\ \text { H29 } & -5.6612847004 & -2.4289308936 & -0.3604517878 \\ \text { C30 } & 5.2071831637 & -1.4398753974 & -0.5103648765 \\ \text { H31 } & 5.5692481759 & -1.0740610169 & -1.4808928419 \\ \text { H32 } & 5.5827745221 & -0.7676234785 & 0.2672792122 \\ \text { H33 } & 5.6612847011 & -2.4289308918 & -0.3604517878\end{array}$

\section{Terminal-TS (Figure 2)}

MNP561 24-Apr-2004

B3LYP/6-31G(d) fully optimised TS +freq

$\begin{array}{llll}\text { C1 } & -2.4213829657 & 1.0415418975 & -1.2580708185 \\ \text { C2 } & -2.4178758068 & 1.0744395777 & 0.1555099244 \\ \text { C3 } & -1.2518514964 & 1.0630569294 & 0.9115259529 \\ \text { C4 } & 0.0349091337 & 1.124269338 & 0.3605906408 \\ \text { C5 } & -0.2564827488 & -0.8560108609 & -1.1374779106 \\ \text { C6 } & -1.5432315835 & -0.7326228908 & -1.6920504752 \\ \text { C7 } & -0.3161124453 & -1.7860931645 & -0.016569344 \\ \text { C8 } & -2.4077263395 & -1.6934525249 & -0.933224875 \\ \text { O9 } & -3.5481721782 & -2.0238131002 & -1.1334761141 \\ \text { O10 } & -1.6763988385 & -2.1731637965 & 0.1343831027 \\ \text { O11 } & 0.5313051003 & -2.2337547809 & 0.7116488307\end{array}$




$\begin{array}{llll}\text { H12 } & -1.6115453796 & 1.5762250593 & -1.7524575947 \\ \text { H13 } & -3.3606533724 & 0.8942004159 & 0.6693025312 \\ \text { H14 } & -1.3347434265 & 0.8669897729 & 1.9790603138 \\ \text { H15 } & 0.1644193258 & 1.575303163 & -0.6190330322 \\ \text { H16 } & 0.6849968258 & -0.5970456422 & -1.5998203478 \\ \text { H17 } & -1.7260213945 & -0.6204881712 & -2.7558815459 \\ \text { C18 } & 1.2277909937 & 0.9333390804 & 1.1397837531 \\ \text { C19 } & 2.4753058763 & 1.2387725244 & 0.6971832093 \\ \text { H20 } & 1.1097230442 & 0.5029676187 & 2.1331166946 \\ \text { H21 } & 2.5888836524 & 1.6619135 & -0.3025515429 \\ \text { C22 } & 3.6846814084 & 1.0475048472 & 1.4606216272 \\ \text { C23 } & 4.9146836036 & 1.3687242241 & 1.0122157967 \\ \text { H24 } & 3.5766259385 & 0.6122066524 & 2.4544551705 \\ \text { H25 } & 5.0080008526 & 1.801082469 & 0.0145248734 \\ \text { C26 } & 6.1880067003 & 1.1778457224 & 1.7750713607 \\ \text { H27 } & 6.714028156 & 2.1327637793 & 1.9128638626 \\ \text { H28 } & 6.007452235 & 0.7411392248 & 2.7624718139 \\ \text { H29 } & 6.8790684217 & 0.5192694187 & 1.2309542014 \\ \text { C30 } & -3.7333973163 & 1.0801524767 & -2.0099966283 \\ \text { H31 } & -4.1849894048 & 2.0777209782 & -1.9322855538 \\ \text { H32 } & -3.5925676906 & 0.8655997736 & -3.0744450072 \\ \text { H33 } & -4.4404525333 & 0.3472023485 & -1.6113946719 \\ & & & \end{array}$

Internal-TS-90-a-90-b (Figure 3) B3LYP/6-31G(d) relaxed TS subject to constraints

\begin{tabular}{|c|c|c|c|}
\hline 1 & -1.1654373685 & 0.8712233288 & -0.88461 \\
\hline 2 & -1.1520902801 & 0.8780906912 & 0.5122796915 \\
\hline 3 & 0.0304050822 & 0.8780939556 & 1.26 \\
\hline 4 & 552 & 36 & 0.6 \\
\hline 5 & 1.199 & -0.9917589966 & -0.5881293 \\
\hline 6 & 471 & -0.9917622624 & -1.3360383 \\
\hline 7 & & -1.957310 & 0.5 \\
\hline 8 & 2965721 & -1.9573154853 & -0.7002803211 \\
\hline 9 & 3827 & -2.3633512731 & -1.0492056932 \\
\hline 10 & 1786 & -2.3940663532 & 9006 \\
\hline 01 & 1.7 & -2.3633408404 & 40143 \\
\hline 1 & 591 & 1.366020831 & -1.3 \\
\hline 13 & -2.0905054431 & 0.7002023258 & 1.033284 \\
\hline 14 & 1404 & 0.700207991 & 2.33 \\
\hline 15 & 9192951 & 1.3660256062 & 009 \\
\hline 16 & 2.19 & -0.7812821326 & -0.5 \\
\hline 1 & 0085 & -0.7812883443 & 33108 \\
\hline 1 & 9173484 & 0.7806718869 & 1.487104 \\
\hline C19 & 3.1 & 1.8464760361 & 1.95 \\
\hline 20 & 0603 & -0.222544924 & 1.7600634372 \\
\hline H2 1 & 2.8 & .8464557937 & .7055753 \\
\hline $\mathrm{C} 22$ & -2.4323542559 & 0.7806581513 & -1.6602704796 \\
\hline C23 & -3.1394723661 & 1.8464585202 & -2.0510808447 \\
\hline H24 & -2.8202559503 & -0.2225606527 & -1.8414437793 \\
\hline $\mathrm{H} 2$ & -2.757669557 & 2.8464403271 & -1.8365920936 \\
\hline $\mathrm{C} 26$ & -4.4483959079 & 1.7812401502 & -2.7837847995 \\
\hline H2 7 & -5.2429758963 & 2.2845426426 & -2.2169023679 \\
\hline H28 & -4.3847139726 & 2.2914530611 & -3.7544207922 \\
\hline H29 & -4.7584952282 & 0.7463783857 & -2.9603594808 \\
\hline $\mathrm{C} 30$ & 4.4205082141 & 1.7812646872 & 2.8278443582 \\
\hline H31 & 5.2703774649 & 2.2914794955 & 2.3546531268 \\
\hline H32 & 4.248480002 & 2.2845691909 & 3.7886343028 \\
\hline H33 & 4.7128680109 & 0.7464045928 & 3.0324645248 \\
\hline
\end{tabular}


Internal-TS-90-a (Figure 3)

B3LYP/6-31G(d) relaxed $T S$ subject to constraints

$\begin{array}{llll}\text { C1 } & -1.2657103483 & 1.0209015505 & -0.8097155991 \\ \text { C2 } & -1.2408742564 & 0.9920141687 & 0.5890027731 \\ \text { C3 } & -0.067562259 & 0.9751510725 & 1.3374601371 \\ \text { C4 } & 1.2118231221 & 0.8915066479 & 0.7454685458 \\ \text { C5 } & 1.1630131513 & -0.8971070803 & -0.2926013129 \\ \text { C6 } & 0.0727382616 & -0.9662741618 & -1.1756006708 \\ \text { C7 } & 0.8602572351 & -1.8558672778 & 0.8134204922 \\ \text { C8 } & -0.9232337311 & -1.8776360518 & -0.6109370116 \\ \text { O9 } & -1.9713954368 & -2.2884726576 & -1.0346320101 \\ \text { O10 } & -0.4449613073 & -2.2862176114 & 0.6593507723 \\ \text { O11 } & 1.5564025635 & -2.2309943854 & 1.7215941801 \\ \text { H12 } & -0.4201911006 & 1.4530618151 & -1.3375807229 \\ \text { H13 } & -2.1842541363 & 0.8398724061 & 1.1104551343 \\ \text { H14 } & -0.1429089002 & 0.8194123022 & 2.4113589081 \\ \text { H15 } & 1.3293979206 & 1.4183862806 & -0.2012911504 \\ \text { H16 } & 2.1946819627 & -0.786020388 & -0.6069577206 \\ \text { H17 } & 0.0707757045 & -0.7275645377 & -2.2292460281 \\ \text { C18 } & 2.4395530849 & 0.8700741035 & 1.5957102169 \\ \text { C19 } & 3.0985096684 & 1.9714659233 & 1.9703623674 \\ \text { H20 } & 2.7437092979 & -0.1019296741 & 1.9841736952 \\ \text { H21 } & 2.7638531903 & 2.9431689947 & 1.6015161552 \\ \text { C22 } & -2.4893106544 & 0.8773448094 & -1.5731194093 \\ \text { C23 } & -2.6008467562 & 1.1997158714 & -2.8755603483 \\ \text { H24 } & -3.3525894684 & 0.4701764211 & -1.0483571739 \\ \text { H25 } & -1.7246516532 & 1.5968069476 & -3.3922558854 \\ \text { C26 } & 4.2972123398 & 1.9848365799 & 2.8745744092 \\ \text { H27 } & 4.5663293742 & 0.9744379157 & 3.1986405215 \\ \text { H28 } & 5.1680680242 & 2.4281599571 & 2.3728941208 \\ \text { H29 } & 4.108951188 & 2.5921656205 & 3.7700183309 \\ \text { C30 } & -3.8448899999 & 1.0572711715 & -3.6946291752 \\ \text { H31 } & -3.6771189853 & 0.384460354 & -4.5467620875 \\ \text { H32 } & -4.6743521519 & 0.6570689584 & -3.1036651956 \\ \text { H33 } & -4.1541399692 & 2.0234299009 & -4.1164569263 \\ & & & \end{array}$

Terminal-TS-90-a-90-b (Figure 3)

B3LYP/6-31G(d) relaxed TS subject to constraints

$\begin{array}{llll}\text { C1 } & -2.3488558319 & 0.9207323689 & -1.5300154743 \\ \text { C2 } & -2.3185966626 & 0.9573417691 & -0.1362703203 \\ \text { C3 } & -1.1315707712 & 0.9475156692 & 0.6032954061 \\ \text { C4 } & 0.1311123306 & 0.8818795206 & 0.0072001998 \\ \text { C5 } & -0.0401204858 & -1.0138705365 & -1.1716182493 \\ \text { C6 } & -1.2270506709 & -1.0029961628 & -1.9124865261 \\ \text { C7 } & -0.2475399452 & -1.9291375036 & -0.0270513232 \\ \text { C8 } & -2.1827871511 & -1.9008455959 & -1.2306231695 \\ \text { O9 } & -3.2817591061 & -2.2715579974 & -1.5490269566 \\ \text { O10 } & -1.5907825896 & -2.3122664156 & -0.0295932981 \\ \text { O11 } & 0.5215806585 & -2.3316632426 & 0.8065820397 \\ \text { H12 } & -1.5150984783 & 1.3690720174 & -2.0642859678 \\ \text { H13 } & -3.2539345259 & 0.8052685207 & 0.4003979059 \\ \text { H14 } & -1.1970743546 & 0.7947873315 & 1.6784640571 \\ \text { H15 } & 0.2427213772 & 1.3470405894 & -0.9702969255 \\ \text { H16 } & 0.9564956267 & -0.8619985142 & -1.5647966499\end{array}$




$\begin{array}{llll}\text { H17 } & -1.3087471465 & -0.8349435613 & -2.9781635738 \\ \text { C18 } & 1.3763589853 & 0.7870242516 & 0.818731228 \\ \text { C19 } & 2.0601642602 & 1.8568989053 & 1.2431832619 \\ \text { H20 } & 1.6941454855 & -0.2118829805 & 1.1172518849 \\ \text { H21 } & 1.6960368421 & 2.854605052 & 0.9831081667 \\ \text { C22 } & 3.2504392712 & 1.7955908794 & 2.1290580563 \\ \text { C23 } & 4.5141554744 & 1.696794503 & 1.7050009407 \\ \text { H24 } & 3.0609631389 & 1.9067633711 & 3.1997671461 \\ \text { H25 } & 4.7019082259 & 1.61312274 & 0.6335962651 \\ \text { C26 } & 5.7223113028 & 1.676542055 & 2.596515029 \\ \text { H27 } & 6.4059461297 & 2.502050486 & 2.3562251486 \\ \text { H28 } & 5.4446471968 & 1.7609690562 & 3.652513786 \\ \text { H29 } & 6.2946715322 & 0.7480836471 & 2.4680961968 \\ \text { C30 } & -3.6391990996 & 0.8364825979 & -2.2993551013 \\ \text { H31 } & -4.3491037662 & 0.157330244 & -1.8193654164 \\ \text { H32 } & -4.1023844624 & 1.8308688539 & -2.3674245313 \\ \text { H33 } & -3.4764305604 & 0.483048063 & -3.3221655203\end{array}$

\section{Terminal-TS-90-b (Figure 3)}

B3LYP/6-31G(d) relaxed TS subject to constraints

$\begin{array}{llll}\text { C1 } & -2.2979220233 & 0.8174359445 & -1.581621016 \\ \text { C2 } & -2.2570882848 & 0.8025823928 & -0.1875006302 \\ \text { C3 } & -1.0647156952 & 0.7756469931 & 0.5430144598 \\ \text { C4 } & 0.194674156 & 0.7454839339 & -0.0637089804 \\ \text { C5 } & 0.0300413706 & -1.105551913 & -1.3252095906 \\ \text { C6 } & -1.1674538186 & -1.077273513 & -2.0479626413 \\ \text { C7 } & -0.1505708981 & -2.0677411506 & -0.216215452 \\ \text { C8 } & -2.1038255448 & -2.0127134523 & -1.3893201488 \\ \text { O9 } & -3.2040322354 & -2.3810470118 & -1.706184695 \\ \text { O10 } & -1.4891824361 & -2.4673824343 & -0.2157940288 \\ \text { O11 } & 0.6348627555 & -2.4946118113 & 0.5901470811 \\ \text { H12 } & -1.4721782101 & 1.2926945996 & -2.1050233467 \\ \text { H13 } & -3.1871875612 & 0.623312366 & 0.3498720455 \\ \text { H14 } & -1.1207886377 & 0.5810925775 & 1.6118979696 \\ \text { H15 } & 0.2901445524 & 1.2534399755 & -1.022290185 \\ \text { H16 } & 1.0192057911 & -0.9213604542 & -1.7229822785 \\ \text { H17 } & -1.2659112789 & -0.8692590717 & -3.1050813548 \\ \text { C18 } & 1.4464046446 & 0.628081932 & 0.7270743151 \\ \text { C19 } & 2.1349819071 & 1.6859988743 & 1.1939160296 \\ \text { H20 } & 1.7559390699 & -0.3802815647 & 1.0038411118 \\ \text { H21 } & 1.8022123064 & 2.6954228631 & 0.9461500083 \\ \text { C22 } & 3.324165774 & 1.5824411027 & 2.0243554166 \\ \text { C23 } & 4.0101300046 & 2.6389135493 & 2.4925038582 \\ \text { H24 } & 3.6588714292 & 0.574501224 & 2.2732903064 \\ \text { H25 } & 3.6618525613 & 3.6414554492 & 2.2372833605 \\ \text { C26 } & 5.2349111961 & 2.5612862092 & 3.3526919241 \\ \text { H27 } & 5.5168785948 & 1.5239693204 & 3.561077783 \\ \text { H28 } & 6.0899709711 & 3.0573576795 & 2.8729335619 \\ \text { H29 } & 5.0787370004 & 3.0713847323 & 4.3133100184 \\ \text { C30 } & -3.5941851635 & 0.7528552214 & -2.3430982367 \\ \text { H31 } & -4.2957542704 & 0.0519633104 & -1.8824299891 \\ \text { H32 } & -4.0643977528 & 1.7458973022 & -2.3710982343 \\ \text { H33 } & -3.438064986 & 0.4380630039 & -3.3796134786 \\ & & & \end{array}$


1k-H-in (Figure 5)

B3LYP/6-31-G(d) fully optimised TS + freq

\begin{tabular}{|c|c|c|c|c|}
\hline $\mathrm{C}$ & 0 & 3.264511 & -1.229400 & 0.725266 \\
\hline $\mathrm{C}$ & 0 & 2.659378 & -0.158936 & 0.056137 \\
\hline $\mathrm{C}$ & 0 & 1.275650 & -0.032593 & -0.098526 \\
\hline $\mathrm{C}$ & 0 & 0.370361 & -0.958817 & 0.416229 \\
\hline $\mathrm{C}$ & 0 & -1.141052 & -0.930740 & 0.218521 \\
\hline $\mathrm{C}$ & 0 & -1.631332 & -0.044174 & -0.970825 \\
\hline $\mathrm{C}$ & 0 & -1.784409 & -0.587708 & 1.562408 \\
\hline $\mathrm{C}$ & 0 & -2.814987 & -0.708237 & -1.670944 \\
\hline $\mathrm{C}$ & 0 & -2.008896 & 1.332535 & -0.406357 \\
\hline 0 & 0 & -3.610321 & -1.453156 & -1.133453 \\
\hline $\mathrm{C}$ & 0 & -2.669846 & 0.390839 & 1.782654 \\
\hline $\mathrm{C}$ & 0 & -2.471359 & 2.485062 & -1.286438 \\
\hline $\mathrm{C}$ & 0 & -3.115463 & 1.256683 & 0.643800 \\
\hline 0 & 0 & -2.198181 & 2.749298 & -2.425751 \\
\hline $\mathrm{O}$ & 0 & -2.884064 & -0.333847 & -2.955185 \\
\hline $\mathrm{C}$ & 0 & -3.409256 & 2.748413 & 0.825745 \\
\hline $\mathrm{C}$ & 0 & -3.971161 & -0.892136 & -3.713303 \\
\hline 0 & 0 & -3.268188 & 3.293228 & -0.511414 \\
\hline $\mathrm{C}$ & 0 & 1.168335 & -2.892014 & -0.555100 \\
\hline $\mathrm{C}$ & 0 & 2.564545 & -2.908798 & -0.438339 \\
\hline $\mathrm{C}$ & 0 & 0.837906 & -2.510315 & -1.938049 \\
\hline $\mathrm{C}$ & 0 & 3.111038 & -2.536469 & -1.769928 \\
\hline $\mathrm{O}$ & 0 & 4.243988 & -2.509705 & -2.169861 \\
\hline $\mathrm{O}$ & 0 & 2.035995 & -2.171326 & -2.58105 \\
\hline $\mathrm{O}$ & 0 & -0.218887 & -2.459702 & -2.513733 \\
\hline $\mathrm{C}$ & 0 & 4.764587 & -1.352386 & 0.811508 \\
\hline $\mathrm{H}$ & 0 & 2.719513 & -1.666183 & 1.559189 \\
\hline $\mathrm{H}$ & 0 & 3.295883 & 0.497833 & -0.534531 \\
\hline $\mathrm{H}$ & 0 & 0.925385 & 0.720978 & -0.798996 \\
\hline $\mathrm{H}$ & 0 & 0.667068 & -1.489120 & 1.317429 \\
\hline $\mathrm{H}$ & 0 & -1.456635 & -1.956924 & -0.010422 \\
\hline $\mathrm{H}$ & 0 & -0.848877 & 0.066254 & -1.723557 \\
\hline $\mathrm{H}$ & 0 & -1.492172 & -1.236328 & 2.387680 \\
\hline $\mathrm{H}$ & 0 & -1.111404 & 1.718292 & 0.105881 \\
\hline $\mathrm{H}$ & 0 & -3.111435 & 0.520462 & 2.769272 \\
\hline $\mathrm{H}$ & 0 & -4.008118 & 0.803879 & 0.185028 \\
\hline $\mathrm{H}$ & 0 & -4.421646 & 2.976883 & 1.165817 \\
\hline $\mathrm{H}$ & 0 & -2.684662 & 3.240036 & 1.487900 \\
\hline $\mathrm{H}$ & 0 & -4.930771 & -0.618622 & -3.266495 \\
\hline $\mathrm{H}$ & 0 & -3.886368 & -1.980728 & -3.747513 \\
\hline $\mathrm{H}$ & 0 & -3.875580 & -0.465668 & -4.711472 \\
\hline $\mathrm{H}$ & 0 & 0.473007 & -3.480530 & 0.028091 \\
\hline $\mathrm{H}$ & 0 & 3.126984 & -3.587784 & 0.190475 \\
\hline $\mathrm{H}$ & 0 & 5.069626 & -2.358032 & 1.117203 \\
\hline $\mathrm{H}$ & 0 & 5.157349 & -0.650162 & 1.559082 \\
\hline $\mathrm{H}$ & 0 & 5.239532 & -1.135345 & -0.149292 \\
\hline
\end{tabular}

\section{1k-H-Ou (Figure 5)}

B3LYP/6-31-G(d) fully optimised TS + freq

$\begin{array}{rrrrr}\text { C } & 0 & 3.264511 & -1.229400 & 0.725266 \\ \text { C } & 0 & 2.659378 & -0.158936 & 0.056137 \\ \text { C } & 0 & 1.275650 & -0.032593 & -0.098526 \\ \text { C } & 0 & 0.370361 & -0.958817 & 0.416229 \\ \text { C } & 0 & -1.141052 & -0.930740 & 0.218521\end{array}$




$\begin{array}{rrrrr}\mathrm{C} & 0 & -1.631332 & -0.044174 & -0.970825 \\ \mathrm{C} & 0 & -1.784409 & -0.587708 & 1.562408 \\ \mathrm{C} & 0 & -2.814987 & -0.708237 & -1.670944 \\ \mathrm{C} & 0 & -2.008896 & 1.332535 & -0.406357 \\ \mathrm{O} & 0 & -3.610321 & -1.453156 & -1.133453 \\ \mathrm{C} & 0 & -2.669846 & 0.390839 & 1.782654 \\ \mathrm{C} & 0 & -2.471359 & 2.485062 & -1.286438 \\ \mathrm{C} & 0 & -3.115463 & 1.256683 & 0.643800 \\ \mathrm{O} & 0 & -2.198181 & 2.749298 & -2.425751 \\ \mathrm{O} & 0 & -2.884064 & -0.333847 & -2.955185 \\ \mathrm{C} & 0 & -3.409256 & 2.748413 & 0.825745 \\ \mathrm{C} & 0 & -3.971161 & -0.892136 & -3.713303 \\ \mathrm{O} & 0 & -3.268188 & 3.293228 & -0.511414 \\ \mathrm{C} & 0 & 1.168335 & -2.892014 & -0.555100 \\ \mathrm{C} & 0 & 2.564545 & -2.908798 & -0.438339 \\ \mathrm{C} & 0 & 0.837906 & -2.510315 & -1.938049 \\ \mathrm{C} & 0 & 3.111038 & -2.536469 & -1.769928 \\ \mathrm{O} & 0 & 4.243988 & -2.509705 & -2.169861 \\ \mathrm{O} & 0 & 2.035995 & -2.171326 & -2.581055 \\ \mathrm{O} & 0 & -0.218887 & -2.459702 & -2.513733 \\ \mathrm{C} & 0 & 4.764587 & -1.352386 & 0.811508 \\ \mathrm{H} & 0 & 2.719513 & -1.666183 & 1.559189 \\ \mathrm{H} & 0 & 3.295883 & 0.497833 & -0.534531 \\ \mathrm{H} & 0 & 0.925385 & 0.720978 & -0.798996 \\ \mathrm{H} & 0 & 0.667068 & -1.489120 & 1.317429 \\ \mathrm{H} & 0 & -1.456635 & -1.956924 & -0.010422 \\ \mathrm{H} & 0 & -0.848877 & 0.066254 & -1.723557 \\ \mathrm{H} & 0 & -1.492172 & -1.236328 & 2.387680 \\ \mathrm{H} & 0 & -1.111404 & 1.718292 & 0.105881 \\ \mathrm{H} & 0 & -3.111435 & 0.520462 & 2.769272 \\ \mathrm{H} & 0 & -4.008118 & 0.803879 & 0.185028 \\ \mathrm{H} & 0 & -4.421646 & 2.976883 & 1.165817 \\ \mathrm{H} & 0 & -2.684662 & 3.240036 & 1.487900 \\ \mathrm{H} & 0 & -4.930771 & -0.618622 & -3.266495 \\ \mathrm{H} & 0 & -3.886368 & -1.980728 & -3.747513 \\ \mathrm{H} & 0 & -3.875580 & -0.465668 & -4.711472 \\ \mathrm{H} & 0 & 3.473007 & -3.480530 & 0.028091 \\ \mathrm{H} & 5.126984 & -3.587784 & 0.190475 \\ \mathrm{H} & 0 & 5.2395326 & -1.135345 & -0.149292\end{array}$

\section{ul-H-in (Figure 5)}

B3LYP/6-31-G(d) fully optimised TS + freq

$\begin{array}{rrrrr}\text { C } & 0 & 0.963242 & 0.061325 & -0.504944 \\ \text { C } & 0 & 2.052094 & 0.154282 & -1.369095 \\ \text { C } & 0 & 3.370667 & -0.106064 & -0.992249 \\ \text { C } & 0 & 3.727493 & -0.531967 & 0.295441 \\ \text { C } & 0 & -0.441711 & 0.350281 & -1.012543 \\ \text { C } & 0 & -1.583221 & -0.382523 & -0.252106 \\ \text { C } & 0 & -0.613937 & 1.872940 & -0.970108 \\ \text { C } & 0 & -2.799713 & -0.538284 & -1.170410 \\ \text { C } & 0 & -1.875290 & 0.418396 & 1.016281 \\ \text { O } & 0 & -2.801132 & -0.365280 & -2.369141 \\ \text { C } & 0 & -1.475952 & 2.543823 & -0.192352 \\ \text { C } & 0 & -2.779251 & -0.056091 & 2.141769 \\ \text { C } & 0 & -2.432068 & 1.801880 & 0.696900 \\ \text { O } & 0 & -3.005371 & -1.165939 & 2.540466\end{array}$




$\begin{array}{rrrrr}\mathrm{O} & 0 & -3.881821 & -0.928574 & -0.470184 \\ \mathrm{C} & 0 & -2.788055 & 2.273087 & 2.110947 \\ \mathrm{C} & 0 & -5.065796 & -1.194595 & -1.244232 \\ \mathrm{O} & 0 & -3.274521 & 1.069046 & 2.759216 \\ \mathrm{C} & 0 & 2.670547 & -2.356232 & 0.443405 \\ \mathrm{C} & 0 & 1.296509 & -2.188600 & 0.222015 \\ \mathrm{C} & 0 & 3.205246 & -3.077469 & -0.747406 \\ \mathrm{C} & 0 & 0.971375 & -2.769104 & -1.087509 \\ \mathrm{O} & 0 & -0.082794 & -2.934219 & -1.645187 \\ \mathrm{O} & 0 & 2.181229 & -3.164688 & -1.684784 \\ \mathrm{O} & 0 & 4.301086 & -3.523865 & -0.958649 \\ \mathrm{C} & 0 & 5.164047 & -0.836655 & 0.643997 \\ \mathrm{H} & 0 & 1.129379 & 0.232712 & 0.555023 \\ \mathrm{H} & 0 & 1.845642 & 0.278610 & -2.431274 \\ \mathrm{H} & 0 & 4.119797 & -0.176575 & -1.779190 \\ \mathrm{H} & 0 & 3.127380 & -0.157074 & 1.122100 \\ \mathrm{H} & 0 & -0.499313 & 0.034180 & -2.059296 \\ \mathrm{H} & 0 & -1.282135 & -1.405121 & -0.010117 \\ \mathrm{H} & 0 & 0.045929 & 2.426758 & -1.634811 \\ \mathrm{H} & 0 & -0.905533 & 0.579132 & 1.518738 \\ \mathrm{H} & 0 & -1.539841 & 3.628600 & -0.260941 \\ \mathrm{H} & 0 & -3.389923 & 1.677277 & 0.166916 \\ \mathrm{H} & 0 & -3.587326 & 3.015411 & 2.159225 \\ \mathrm{H} & 0 & -1.913986 & 2.645340 & 2.661102 \\ \mathrm{H} & 0 & -5.385574 & -0.295425 & -1.777359 \\ \mathrm{H} & 0 & -4.872620 & -1.989929 & -1.968231 \\ \mathrm{H} & 0 & -5.819611 & -1.505035 & -0.521149 \\ \mathrm{H} & 0 & 3.112000 & -2.552841 & 1.413300 \\ \mathrm{H} & 0 & 0.542572 & -2.093109 & 0.990784 \\ \mathrm{H} & 0 & 5.242874 & -1.373291 & 1.594742 \\ \mathrm{H} & 0 & 5.731888 & 0.097641 & 0.747559 \\ \mathrm{H} & 0 & 5.640228 & -1.447449 & -0.128128 \\ & & & & \end{array}$

\section{ul-H-Ou (Figure 5)}

B3LYP/6-31-G(d) fully optimised TS + freq

$\begin{array}{rrrrr}\text { C } & 0 & 0.218002 & -0.958817 & -0.606644 \\ \text { C } & 0 & 0.866684 & -0.093613 & -1.485535 \\ \text { C } & 0 & 2.227280 & -0.196732 & -1.784668 \\ \text { C } & 0 & 3.056717 & -1.183731 & -1.233994 \\ \text { C } & 0 & -1.271820 & -0.907104 & -0.299011 \\ \text { C } & 0 & -1.497772 & -0.280700 & 1.126678 \\ \text { C } & 0 & -2.087418 & -0.223679 & -1.388840 \\ \text { C } & 0 & -2.613190 & -1.029739 & 1.861679 \\ \text { C } & 0 & -1.686493 & 1.218628 & 0.941498 \\ \text { O } & 0 & -2.755493 & -2.232910 & 1.812088 \\ \text { C } & 0 & -2.818268 & 0.890000 & -1.238007 \\ \text { C } & 0 & -1.792923 & 2.196548 & 2.097595 \\ \text { C } & 0 & -2.909058 & 1.567108 & 0.098450 \\ \text { O } & 0 & -1.332000 & 2.138596 & 3.205978 \\ \text { O } & 0 & -3.380615 & -0.219070 & 2.611069 \\ \text { C } & 0 & -2.900543 & 3.091600 & 0.258316 \\ \text { C } & 0 & -4.386230 & -0.871307 & 3.411179 \\ \text { O } & 0 & -2.489563 & 3.287064 & 1.638351 \\ \text { C } & 0 & 2.106598 & -2.979324 & -1.902328 \\ \text { C } & 0 & 0.740067 & -2.992264 & -1.581680 \\ \text { C } & 0 & 2.199783 & -2.780900 & -3.373627 \\ \text { C } & 0 & -0.012863 & -2.796890 & -2.831223 \\ \text { O } & 0 & -1.194794 & -2.841873 & -3.058914\end{array}$




$\begin{array}{rrrrr}\mathrm{O} & 0 & 0.913437 & -2.527008 & -3.848450 \\ \mathrm{O} & 0 & 3.152084 & -2.802567 & -4.106415 \\ \mathrm{C} & 0 & 4.511200 & -1.286850 & -1.619019 \\ \mathrm{H} & 0 & 0.803375 & -1.405685 & 0.192490 \\ \mathrm{H} & 0 & 0.257446 & 0.575653 & -2.088516 \\ \mathrm{H} & 0 & 2.613388 & 0.390060 & -2.616791 \\ \mathrm{H} & 0 & 2.829315 & -1.509200 & -0.220993 \\ \mathrm{H} & 0 & -1.622742 & -1.944992 & -0.233215 \\ \mathrm{H} & 0 & -0.605453 & -0.459000 & 1.742767 \\ \mathrm{H} & 0 & -2.076050 & -0.733063 & -2.348724 \\ \mathrm{H} & 0 & -0.803146 & 1.564056 & 0.376572 \\ \mathrm{H} & 0 & -3.412796 & 1.265564 & -2.069351 \\ \mathrm{H} & 0 & -3.807541 & 1.209290 & 0.622238 \\ \mathrm{H} & 0 & -3.874161 & 3.570923 & 0.138245 \\ \mathrm{H} & 0 & -2.171524 & 3.579132 & -0.401947 \\ \mathrm{H} & 0 & -5.087982 & -1.412964 & 2.772186 \\ \mathrm{H} & 0 & -3.918472 & -1.572235 & 4.106415 \\ \mathrm{H} & 0 & -4.889435 & -0.070145 & 3.951477 \\ \mathrm{H} & 0 & 2.860107 & -3.579117 & -1.406769 \\ \mathrm{H} & 0 & 0.284943 & -3.512680 & -0.749756 \\ \mathrm{H} & 0 & 4.648788 & -1.180145 & -2.698441 \\ \mathrm{H} & 0 & 4.940536 & -2.247955 & -1.319229 \\ \mathrm{H} & 0 & 5.087982 & -0.499512 & -1.115356\end{array}$

\section{Me-an (Figure 6a)}

maleic anhydride + 1-Ethylbutadiene endo TS fully optimised B3LYP/6-31G(d)

$\begin{array}{llll}\text { C1 } & 2.1599343719 & 0.8986225825 & 0.2894686111 \\ \text { C2 } & 1.3629624605 & 1.3676613812 & -0.7536923121 \\ \text { C3 } & -0.0174368608 & 1.5731578889 & -0.6122443873 \\ \text { C4 } & -0.6999206117 & 1.3698525336 & 0.5778478627 \\ \text { C5 } & -2.197868123 & 1.4565995407 & 0.6826080681 \\ \text { C6 } & -2.6605675617 & 2.8462306773 & 1.1628795157 \\ \text { H7 } & 1.9547514519 & 1.2072526712 & 1.30992519 \\ \text { H8 } & 3.1997805885 & 0.6539879245 & 0.0911808608 \\ \text { H9 } & 1.7723661447 & 1.3544779865 & -1.7613395101 \\ \text { H10 } & -0.6042009077 & 1.7207125493 & -1.5177357426 \\ \text { H11 } & -0.1557937654 & 1.4783482878 & 1.5129437343 \\ \text { H12 } & -2.5643559442 & 0.6938291236 & 1.3799014574 \\ \text { H13 } & -2.6495227405 & 1.222641228 & -0.2874907903 \\ \text { H14 } & -2.2364797825 & 3.0918441224 & 2.1436991595 \\ \text { H15 } & -3.7519090968 & 2.8751689428 & 1.2524985545 \\ \text { H16 } & -2.3552676265 & 3.6296721142 & 0.4607872984 \\ \text { C17 } & -0.0418265636 & -0.9105524018 & 0.9876845052 \\ \text { C18 } & 1.3297320804 & -1.0060570431 & 0.7420443091 \\ \text { C19 } & -0.7542043367 & -1.432095318 & -0.1859028801 \\ \text { C20 } & 1.4810583033 & -1.6428358628 & -0.5991417418 \\ \text { O21 } & 2.46828618 & -2.0191995546 & -1.1702308538 \\ \text { O22 } & 0.2143727203 & -1.7399015444 & -1.1630180315 \\ \text { O23 } & -1.9251986886 & -1.6174568729 & -0.3894413689 \\ \text { H24 } & -0.5272737966 & -0.8549689823 & 1.9520264622 \\ \text { H25 } & 2.0870448293 & -1.1840060632 & 1.495816055\end{array}$


Me-in (Figure 6a)

maleic anhydride + 1-Ethylbutadiene

endo TS fully optimised B3LYP/6-31G(d)

$\begin{array}{llcc}\text { C1 } & 0.4772141872 & 1.9333267482 & -1.4102576764 \\ \text { C2 } & -0.8125535136 & 1.7324610539 & -0.9088732749 \\ \text { C3 } & -1.0611919119 & 1.3444592388 & 0.4164655484 \\ \text { C4 } & -0.0627604689 & 1.1885484498 & 1.3633418165 \\ \text { C5 } & -0.2658133893 & 0.6218317468 & 2.7454544564 \\ \text { C6 } & -1.6528072225 & 0.0484758591 & 3.0481023221 \\ \text { H7 } & 1.2331295926 & 2.3890673022 & -0.7768292236 \\ \text { H8 } & 0.5929780724 & 2.1512311261 & -2.4687238085 \\ \text { H9 } & -1.6315109123 & 1.6495227893 & -1.6199235518 \\ \text { H10 } & -2.0633861643 & 1.0002905203 & 0.6570828978 \\ \text { H11 } & 0.8661258737 & 1.7342056017 & 1.2247903551 \\ \text { H12 } & 0.4834194651 & -0.1641970785 & 2.9093708179 \\ \text { H13 } & -0.0203502554 & 1.4148164343 & 3.4692168978 \\ \text { H14 } & -1.8909835395 & -0.7817656829 & 2.3765033491 \\ \text { H15 } & -2.4367390513 & 0.8092603835 & 2.9589082362 \\ \text { H16 } & -1.6803290152 & -0.3366219835 & 4.0724503188 \\ \text { C17 } & 1.3833069878 & -0.3729864676 & 0.0777109061 \\ \text { C18 } & 1.3980918592 & 0.0980885578 & -1.2396630885 \\ \text { C19 } & 0.4031506682 & -1.4520472398 & 0.1742187878 \\ \text { C20 } & 0.434585613 & -0.7593706353 & -2.0009336715 \\ \text { O21 } & 0.2035730325 & -0.7966147921 & -3.1789184259 \\ \text { O22 } & -0.2300397192 & -1.5607607706 & -1.0857952495 \\ \text { O23 } & 0.1092749791 & -2.1889223592 & 1.0809452012 \\ \text { H24 } & 2.1532137725 & -0.2466232793 & 0.8258330766 \\ \text { H25 } & 2.2846289667 & 0.4544733716 & -1.7519283292\end{array}$

\section{Me-ou (Figure 6a)}

maleic anhydride + 1-Ethylbutadiene

endo TS fully optimised B3LYP/6-31G(d)

$\begin{array}{llcc}\text { C1 } & 1.2549095358 & 1.9984159367 & 0.5898024374 \\ \text { C2 } & 0.7531535727 & 1.9635544972 & -0.7115418724 \\ \text { C3 } & -0.5310218544 & 1.4880096898 & -1.014559905 \\ \text { C4 } & -1.4405253657 & 1.0677788517 & -0.057355895 \\ \text { C5 } & -2.7651014863 & 0.4498654798 & -0.420147522 \\ \text { C6 } & -3.4346349124 & -0.3602699949 & 0.6959381698 \\ \text { H7 } & 0.5932381475 & 2.230540222 & 1.4188499226 \\ \text { H8 } & 2.290844843 & 2.2868524384 & 0.7455220504 \\ \text { H9 } & 1.4518772611 & 2.0907046144 & -1.5353994422 \\ \text { H10 } & -0.7609260017 & 1.2848329593 & -2.0595207395 \\ \text { H11 } & -1.3586353468 & 1.4627098289 & 0.9521939599 \\ \text { H12 } & -2.6426946301 & -0.1780646267 & -1.3105256852 \\ \text { H13 } & -3.4356210553 & 1.2744941146 & -0.7137138699 \\ \text { H14 } & -3.4954130204 & 0.2136908715 & 1.6292719252 \\ \text { H15 } & -2.8854164986 & -1.2866925559 & 0.8844331874 \\ \text { H16 } & -4.4554468529 & -0.6329691643 & 0.4090318969 \\ \text { C17 } & 0.0068503968 & -0.6041036958 & 1.0428636948 \\ \text { C18 } & 1.2690014929 & -0.0216742072 & 1.1800541798 \\ \text { C19 } & 0.0228835858 & -1.4633842291 & -0.1431632592 \\ \text { C20 } & 2.1163978566 & -0.5825513454 & 0.0847183269 \\ \text { O21 } & 3.2932553955 & -0.4608220827 & -0.1213406687 \\ \text { O22 } & 1.2872839759 & -1.3180601334 & -0.7522483196 \\ \text { O23 } & -0.8066647948 & -2.2039793148 & -0.6062828659 \\ \text { H24 } & -0.7401863034 & -0.7247102325 & 1.8136543019 \\ \text { H25 } & 1.7359059134 & 0.2276578813 & 2.1255271956\end{array}$




\section{H-an (Figure 6b)}

maleic anhydride + 1-iso-Propylbutadiene endo TS fully optimised B3LYP/6-31G(d)

$\begin{array}{llll}\text { C1 } & 0.9225069271 & 1.9390641593 & -1.3836475345 \\ \text { C2 } & -0.4652300521 & 1.8075407738 & -1.2527461708 \\ \text { C3 } & -1.0817899384 & 1.4105861719 & -0.0565407611 \\ \text { C4 } & -0.3886328839 & 1.1852924661 & 1.1203427504 \\ \text { C5 } & -1.0068890023 & 0.7228022548 & 2.4222257443 \\ \text { C6 } & -2.3574568151 & 0.0072389408 & 2.2714692262 \\ \text { C7 } & -0.0272093067 & -0.1178280536 & 3.2637101428 \\ \text { H8 } & 1.4986573144 & 2.3388769533 & -0.553613928 \\ \text { H9 } & 1.3256213337 & 2.1869333807 & -2.3621382222 \\ \text { H10 } & -1.066841585 & 1.7941333042 & -2.1588685296 \\ \text { H11 } & -2.133114936 & 1.1402078897 & -0.1028390729 \\ \text { H12 } & 0.5916338725 & 1.6413869724 & 1.2324012372 \\ \text { H13 } & -1.1805145638 & 1.657271532 & 2.986744727 \\ \text { H14 } & -2.2538146627 & -0.9014533808 & 1.6705298635 \\ \text { H15 } & -3.1151191385 & 0.6494214595 & 1.8095255726 \\ \text { H16 } & -2.7350140797 & -0.282580981 & 3.2579823029 \\ \text { H17 } & 0.9445675045 & 0.3809645519 & 3.3678904741 \\ \text { H18 } & 0.1266092007 & -1.1002753465 & 2.8074901231 \\ \text { H19 } & -0.4307092864 & -0.2694239899 & 4.2707117508 \\ \text { C20 } & 1.2608054938 & -0.4945026561 & 0.1275744897 \\ \text { C21 } & 1.6647026367 & 0.0715375479 & -1.0891458861 \\ \text { C22 } & 0.2481798509 & -1.5098575722 & -0.1498144562 \\ \text { C23 } & 0.9283760117 & -0.6738434913 & -2.1610228744 \\ \text { O24 } & 1.0462907893 & -0.6177821288 & -3.3548554874 \\ \text { O25 } & -0.0037366812 & -1.4892648483 & -1.5415010389 \\ \text { O26 } & -0.3219799943 & -2.2973659624 & 0.5631266453 \\ \text { H27 } & 1.7990738959 & -0.4788992645 & 1.0634985543 \\ \text { H28 } & 2.6801946914 & 0.3905571864 & -1.2979038266\end{array}$

\section{H-in (Figure 6b)}

maleic anhydride +1 -iso-Propylbutadiene endo TS fully optimised B3LYP/6-31G(d)

$\begin{array}{llll}\text { C1 } & -1.2160713341 & -2.064737099 & 0.6972808125 \\ \text { C2 } & -0.6521576896 & -2.0130811674 & -0.5789170954 \\ \text { C3 } & 0.5436976374 & -1.3320464582 & -0.8478419463 \\ \text { C4 } & 1.3073353369 & -0.7022228336 & 0.1221219443 \\ \text { C5 } & 2.5498481949 & 0.0877522573 & -0.2162942917 \\ \text { C6 } & 2.8913175874 & 1.1584892208 & 0.8310849649 \\ \text { C7 } & 3.7306388324 & -0.8975466222 & -0.3914285841 \\ \text { H8 } & -0.5713292547 & -2.1325297987 & 1.5685373385 \\ \text { H9 } & -2.1897699253 & -2.530915814 & 0.8211213658 \\ \text { H10 } & -1.2669488214 & -2.3098843675 & -1.4256994997 \\ \text { H11 } & 0.7996079579 & -1.1533399075 & -1.8913871396 \\ \text { H12 } & 1.2323647399 & -1.0426018583 & 1.1524837994 \\ \text { H13 } & 2.3807988396 & 0.5912037387 & -1.177015784 \\ \text { H14 } & 2.9650230392 & 0.7226799392 & 1.8365615624 \\ \text { H15 } & 2.1404612003 & 1.9523227539 & 0.8388532654 \\ \text { H16 } & 3.8590578325 & 1.617499357 & 0.6007611944 \\ \text { H17 } & 3.5221605711 & -1.6416079751 & -1.1672255411 \\ \text { H18 } & 3.938399533 & -1.4333701042 & 0.5430183545 \\ \text { H19 } & 4.6378948518 & -0.3511270014 & -0.6738459026\end{array}$




$\begin{array}{llll}\text { C20 } & -0.4981080916 & 0.7394311673 & 1.0278957686 \\ \text { C21 } & -1.632001863 & -0.0685503285 & 1.1517752301 \\ \text { C22 } & -0.6173476818 & 1.511491682 & -0.2112718928 \\ \text { C23 } & -2.5149447608 & 0.2592729513 & -0.0088390701 \\ \text { O24 } & -3.6361202627 & -0.0966453044 & -0.2508714968 \\ \text { O25 } & -1.7983955202 & 1.0900501672 & -0.8592065854 \\ \text { O26 } & 0.0747459974 & 2.3738853989 & -0.6889828768 \\ \text { H27 } & 0.1697104646 & 1.0464342525 & 1.8191544132 \\ \text { H28 } & -2.0925097522 & -0.3426019263 & 2.0937752066\end{array}$

\section{H-Ou (Figure 6b)}

maleic anhydride + 1-iso-Propylbutadiene endo TS fully optimised B3LYP/6-31G(d)

$\begin{array}{llll}\text { C1 } & -1.2621557844 & -2.1633474574 & 0.2330132833 \\ \text { C2 } & -0.5993361682 & -1.8395882934 & -0.9549746882 \\ \text { C3 } & 0.6106678276 & -1.1291046299 & -0.9814624429 \\ \text { C4 } & 1.2877967396 & -0.7402476425 & 0.1623001048 \\ \text { C5 } & 2.5155580522 & 0.1429479628 & 0.1972323329 \\ \text { C6 } & 3.7101693838 & -0.6577401095 & 0.7599558063 \\ \text { C7 } & 2.8646017237 & 0.7990734778 & -1.1448385911 \\ \text { H8 } & -0.6842016526 & -2.4467946531 & 1.1081786268 \\ \text { H9 } & -2.2358633057 & -2.6418499546 & 0.1683600851 \\ \text { H10 } & -1.1464970373 & -1.9374626596 & -1.889995586 \\ \text { H11 } & 0.9373258141 & -0.7356012753 & -1.9400501583 \\ \text { H12 } & 1.116224634 & -1.3061883089 & 1.0741503283 \\ \text { H13 } & 2.301213058 & 0.956000025 & 0.9057570491 \\ \text { H14 } & 3.4830341921 & -1.0883265281 & 1.7425611784 \\ \text { H15 } & 4.584771465 & -0.0075828633 & 0.87285666 \\ \text { H16 } & 3.9838938538 & -1.4793105333 & 0.0873862696 \\ \text { H17 } & 2.0357847634 & 1.4067811338 & -1.519448221 \\ \text { H18 } & 3.1277726919 & 0.0514454781 & -1.9033206331 \\ \text { H19 } & 3.7290742595 & 1.459661141 & -1.0201057068 \\ \text { C20 } & -0.591160108 & 0.4446710215 & 1.2837313573 \\ \text { C21 } & -1.7268779104 & -0.3491453699 & 1.0888505306 \\ \text { C22 } & -0.5965111757 & 1.514535829 & 0.2887494068 \\ \text { C23 } & -2.494488296 & 0.2865139548 & -0.0290741131 \\ \text { O24 } & -3.582369584 & 0.0207845012 & -0.4629972076 \\ \text { O25 } & -1.7077751342 & 1.2973898028 & -0.5586918761 \\ \text { O26 } & 0.1312394059 & 2.460633411 & 0.1251288966 \\ \text { H27 } & 0.0123668966 & 0.5139941355 & 2.1777327336 \\ \text { H28 } & -2.2832428406 & -0.8266393177 & 1.8875209507\end{array}$




\section{Experimental Section}

\section{General Methods}

NMR spectra were recorded at $298 \mathrm{~K}$, at $500 \mathrm{MHz}, 400 \mathrm{MHz}$, or $300 \mathrm{MHz}$. Residual acetonitrile ( $\delta$ $1.96 \mathrm{ppm}$ ), chloroform ( $\delta 7.26 \mathrm{ppm})$, and DMSO ( $\delta 2.51 \mathrm{ppm})$ were used as internal references for ${ }^{1} \mathrm{H}$ NMR spectra measured in these solvents. Residual acetonitrile ( $\delta 1.3 \mathrm{ppm}, 118.3 \mathrm{ppm})$, chloroform ( $\delta$ $77.1 \mathrm{ppm}$ ), and DMSO ( $\delta 39.5 \mathrm{ppm}$ ) were used as internal references for ${ }^{13} \mathrm{C}$ NMR spectra. Assignment of proton signals was assisted by ${ }^{1} \mathrm{H}-{ }^{1} \mathrm{H}$ COSY and NOESY experiments when necessary; assignment of carbon signals was assisted by DEPT experiments. IR spectra were recorded as neat films on $\mathrm{NaCl}$ plates for oils or as $\mathrm{KBr}$ pellets for solid products. Low resolution mass spectra were recorded using electron impact (EI) ionization mode at 40 or $70 \mathrm{eV}$. Melting points are uncorrected. Analytical TLC was performed with silica gel plates, precoated with silica gel 60 F254 $(0.2 \mathrm{~mm})$. Flash chromatography employed 230-400 mesh silica gel.

Reactions were conducted under a positive pressure of dry argon or nitrogen. Benzene, diethyl ether, toluene and THF were dried over sodium wire and distilled from sodium benzophenone ketyl. Dichloromethane was distilled from calcium hydride. Chlorobenzene was purified by the method of Perrin and Armarego. ${ }^{1}$ Commercially available chemicals were purified by standard procedures or used as purchased.

$2 E, 4 E, 6 E, 8 E$-Decatetraen-1-ol (1). To a stirred solution of $2 E, 4 E, 6 E$-octatrienal ${ }^{2}(14.74 \mathrm{~g}, 0.12 \mathrm{~mol})$ in dichloromethane $(200 \mathrm{~mL})$ under Ar was added (carbomethoxymethylene)triphenylphosphorane $(44.40 \mathrm{~g}, 0.13 \mathrm{~mol})$. The resulting mixture was stirred at room temperature for $1 \mathrm{~h}$. After removal of the solvent in vacuo the crude residue was purified on silica gel (300 g) eluting with hexane/ethyl acetate (1:1) to give methyl $2 E, 4 E, 6 E, 8 E$-decatetraenoate $(21.08 \mathrm{~g}, 0.12 \mathrm{~mol}, 98 \%)$ as a pale yellow solid: $\mathrm{mp}$ $121{ }^{\circ} \mathrm{C}$ dec. $(\mathrm{THF} / \mathrm{hexane}) ; \mathrm{R}_{\mathrm{f}}=0.34$; hexane/ethyl acetate $(1: 1) ;{ }^{1} \mathrm{H}$ NMR $\left(300 \mathrm{MHz}, \mathrm{CDCl}_{3}\right): \delta 7.31$ (1H, dd $J=15.3,11.3 \mathrm{~Hz}, \mathrm{H} 3), 6.56$ (1H, dd, $J=14.8,11.0 \mathrm{~Hz}, \mathrm{H} 5), 6.08-6.42(4 \mathrm{H}, \mathrm{m} \mathrm{H} 4, \mathrm{H} 6, \mathrm{H} 7$, H8), $5.85(1 \mathrm{H}, \mathrm{d}, J=15.3 \mathrm{~Hz}, \mathrm{H} 2), 5.68(1 \mathrm{H}, \mathrm{dq}, J=14.8,6.9 \mathrm{~Hz}, \mathrm{H} 9), 3.74\left(3 \mathrm{H}, \mathrm{s},-\mathrm{CO}_{2} \mathrm{CH}_{3}\right)$ and 1.81 
$(3 \mathrm{H}, \mathrm{d}, J=6.9 \mathrm{~Hz}, \mathrm{H} 10) \mathrm{ppm} ;{ }^{13} \mathrm{C} \mathrm{NMR}\left(75 \mathrm{MHz}, \mathrm{CDCl}_{3}\right): \delta 168.0,145.2,141.5,138.0,133.5,132.0$, 129.7, 129.4, 120.0, 51.8 and 18.9 ppm; IR (KBr disc): $v_{\max } 3015,2956,1710,1431 \mathrm{~cm}^{-1}$; MS (70 eV, EI) $m / z(\%): 178(18),[M]^{+} 147$ (51), 74 (100), 104 (12); HRMS: calcd for $\mathrm{C}_{11} \mathrm{H}_{14} \mathrm{O}_{2}[M]^{+}$: 178.0994; found 178.0994 .

To a stirred solution of methyl $2 E, 4 E, 6 E, 8 E$-decatetraenoate $(4.42 \mathrm{~g}, 24.8 \mathrm{mmol})$ in THF $(60 \mathrm{~mL})$ under $\mathrm{Ar}$ at $0{ }^{\circ} \mathrm{C}$ was added dropwise diisobutylaluminium hydride $(1.5 \mathrm{M}$ in toluene, $41.3 \mathrm{~mL}, 62.0$ mmol). Following addition, unreacted diisobutylaluminium hydride was quenched at $0{ }^{\circ} \mathrm{C}$ by the addition of ethyl acetate $(2.0 \mathrm{~mL})$ and the solution left to warm to room temperature. The reaction mixture was partitioned against sat. aq. potassium sodium tartrate $(100 \mathrm{~mL})$ and the aqueous layer extracted with further dichloromethane $(3$ x $40 \mathrm{~mL})$. The combined organic extracts were washed with sat. aq. $\mathrm{NaCl}(60 \mathrm{~mL})$, dried over $\mathrm{MgSO}_{4}$ and concentrated in vacuo to give a pale yellow solid. Purification of this material on silica gel (100 g) eluting with hexane/ethyl acetate (1:1) afforded tetraenol 1 (3.43 g, $22.80 \mathrm{mmol}, 92 \%)$ as a white solid: $\mathrm{mp} 178-181{ }^{\circ} \mathrm{C} \mathrm{dec}$. $(\mathrm{THF}) ; \mathrm{R}_{\mathrm{f}}=0.23$; hexane/ethyl acetate $(1: 1) ;{ }^{1} \mathrm{H}$ NMR $\left(300 \mathrm{MHz}, \mathrm{d}_{6}\right.$-DMSO) $\delta$ 6.05-6.35 (6H, complex m, H3, H4, H5, H6, H7, H8), 5.65-5.88 (2H, m, H2, H9), 4.75 (1H, br. t, $J=5.5 \mathrm{~Hz},-\mathrm{OH}), 4.00$ (2H, br. t, $J=5.2 \mathrm{~Hz}$, $\mathrm{H} 1)$ and $1.74\left(3 \mathrm{H}, \mathrm{d}, J=6.6 \mathrm{~Hz},-\mathrm{CH}_{3}\right) \mathrm{ppm} ;{ }^{13} \mathrm{C} \mathrm{NMR}\left(75 \mathrm{MHz}, \mathrm{d}_{6}\right.$-DMSO) $\delta 134.4,132.8,132.0$, 131.9 [2 coincident resonances], 130.4, 129.7, 129.3, 61.2 and 18.1 ppm; IR ( $\mathrm{KBr}$ disc): $v=3286,2929$, 1640, $1443 \mathrm{~cm}^{-1}$; MS (70 eV, EI): $m / z(\%): 150$ (8) [M] $]^{+}, 149$ (12), 132 (46), 119 (34), 31 (100); HRMS: calc for $\mathrm{C}_{10} \mathrm{H}_{14} \mathrm{O}[M]^{+}:$150.1045; found 150.1021 .

Methyl 2E,4E,6E,8E-decatetraen-1-yl 2Z-butendioate (3). To a stirred solution of tetraenol 1 $(0.20 \mathrm{~g}, 1.85 \mathrm{mmol})$ in dichloromethane $(10 \mathrm{~mL})$ at $0{ }^{\circ} \mathrm{C}$ under $\mathrm{Ar}$ was added triethylamine $(0.30 \mathrm{~mL}$, $2.16 \mathrm{mmol})$, maleic anhydride $(0.30 \mathrm{~g}, 3.04 \mathrm{mmol})$ and 4-DMAP $(16.5 \mathrm{mg}, 0.14 \mathrm{mmol})$. The resulting solution was allowed to warm to room temperature and stirring was continued for 15 min. The reaction mixture was diluted with dichloromethane and washed successively with $10 \%$ aqueous $\mathrm{HCl}(10 \mathrm{~mL})$, water $(10 \mathrm{~mL})$ and satd aq $\mathrm{NaCl}(10 \mathrm{~mL})$. The organic layer was dried over $\mathrm{MgSO}_{4}$ and the solvent was removed in vacuo to give the crude acid as an orange oil. The crude acid was taken up in dichloromethane $(15 \mathrm{~mL})$ and the resulting solution was cooled to $-78{ }^{\circ} \mathrm{C}$. An ethereal solution of 
diazomethane was added dropwise until TLC analysis indicated the complete consumption of the acid starting material. The solvent was removed under reduced pressure and the crude material purified on silica gel (20 g) eluting with hexane/ethyl acetate (2:1) to afford maleate ester 3 (0.40 g, 1.52 mmol, $82 \%$ ) as a pale yellow solid: $\mathrm{mp} 154-156{ }^{\circ} \mathrm{C} \mathrm{dec}$. (hexane/THF); $\mathrm{R}_{\mathrm{f}}=0.18$; hexane/ethyl acetate $(2: 1)$; ${ }^{1} \mathrm{H}$ NMR (300 MHz, $\mathrm{CDCl}_{3}$ ): $\delta 6.25$ (2H, s, H2', H3'), 6.05-6.40 (6H, complex m, H3, H4, H5, H6, H7, H8), 5.70-5.82 (2H, m, H2, H9), $4.72(2 \mathrm{H}, \mathrm{dd}, J=6.9,0.9 \mathrm{~Hz}, \mathrm{H} 1), 3.77\left(3 \mathrm{H}, \mathrm{s},-\mathrm{CO}_{2} \mathrm{CH}_{3}\right)$ and 1.79 $(3 \mathrm{H}, \mathrm{dd}, J=6.1,1.0 \mathrm{~Hz}, \mathrm{H} 10) \mathrm{ppm} ;{ }^{13} \mathrm{C} \mathrm{NMR}\left(75 \mathrm{MHz}, \mathrm{CDCl}_{3}\right): \delta 165.6,164.8,135.4,134.8,134.4$, 131.7, 130.9, 130.3, 129.8 [two coincident resonances], 129.6, 125.0, 65.6, 52.1 and 18.3 ppm; IR $(\mathrm{KBr}$ disc): $v_{\max } 3014,1743,1724,1435 \mathrm{~cm}^{-1}$; MS (70 eV, EI) $m / z(\%): 262(8)[M]^{+}, 231(21), 183(100), 178$ (23), 149 (45), 31 (65); HRMS: calcd for $\mathrm{C}_{15} \mathrm{H}_{18} \mathrm{O} 4[M]^{+}:$262.1205; found 262.1199.

Intramolecular Diels-Alder-Intermolecular Diels-Alder Sequence. A stirred solution of methyl $2 E, 4 E, 6 E, 8 E$-decatetraen-1-yl 2Z-butendioate $(3)(0.15 \mathrm{~g}, 0.58 \mathrm{mmol})$ and BHT (12.7 $\mathrm{mg}, 58 \mu \mathrm{mol})$ in toluene $(120 \mathrm{~mL})$ was heated at reflux for $1 \mathrm{~h}$ at which time TLC indicated complete consumption of starting material. An aliquot of the reaction mixture was removed and ${ }^{1} \mathrm{H}$ NMR analysis, after evacuation of the solvent in vacuo, showed a single product $\mathbf{4}$, which could be readily isolated in pure form by chromatography (see below). To the reaction mixture was added maleic anhydride (0.28 g, 2.90 mmol) and the resulting solution refluxed for a further $8 \mathrm{~h}$. After removal of the solvent under reduced pressure, purification of the crude residue was performed over silica gel ( $20 \mathrm{~g})$ eluting with hexane ethyl acetate (1:1) to afford the double cycloadducts 5 and $\mathbf{6}(0.19 \mathrm{~g}, 0.54 \mathrm{mmol}, 92 \%, \mathbf{5 : 6}=70: 30)$.

\section{Bicycle 4 (Methyl rel 5-[1'E,3'E-butadien-1-yl]-3-oxo-1,3,3aR,4S,5R,7aS-hexahydro-4-iso-}

benzofurancarboxylate). In one run, following the heating of a solution of $3(0.150 \mathrm{~g}, 0.577 \mathrm{mmol})$ and BHT $(13.0 \mathrm{mg}, 59.0 \mu \mathrm{mol})$ in toluene $(115 \mathrm{~mL})$ for $1 \mathrm{~h}$, chromatography on silica gel $(20 \mathrm{~g})$ eluting with hexane/ethyl acetate (1:1) gave, exclusively, IMDA adduct 4 (0.13 g, $0.51 \mathrm{mmol}, 89 \%)$ : colorless oil; $\mathrm{R}_{\mathrm{f}}=0.33$; hexane/ethyl acetate $(1: 1) ;{ }^{1} \mathrm{H} \mathrm{NMR}\left(300 \mathrm{MHz}, \mathrm{CDCl}_{3}\right): \delta 5.95-6.08\left(2 \mathrm{H}, \mathrm{m}, \mathrm{H} 2 ', \mathrm{H} 3{ }^{\prime}\right)$, $5.90(1 \mathrm{H}, \mathrm{ddd}, J=9.9,1.9,1.9 \mathrm{~Hz}, \mathrm{H} 7), 5.55-5.73\left(3 \mathrm{H}, \mathrm{m}, \mathrm{H} 1^{\prime}, \mathrm{H} 4{ }^{\prime}, \mathrm{H} 6\right), 4.50$ (1H, dd, J = 7.6, 7.6 Hz, H1), $3.85(1 \mathrm{H}, \mathrm{dd}, J=11.5,7.9 \mathrm{~Hz}, \mathrm{H} 1), 3.70\left(3 \mathrm{H}, \mathrm{s},-\mathrm{CO}_{2} \mathrm{CH}_{3}\right), 3.53-3.58(1 \mathrm{H}, \mathrm{m}, \mathrm{H} 5), 3.10-3.22$ 
(1H, m [partially obscured], H7a), 3.07-3.10 (1H, m, H4), 2.35 (1H, dd, J = 13.6, 3.3 Hz, H3a) and 0.85 $(3 \mathrm{H}, \mathrm{d}, J=6.2 \mathrm{~Hz}, \mathrm{H} 5) \mathrm{ppm} ;{ }^{13} \mathrm{C} \mathrm{NMR}\left(75 \mathrm{MHz}, \mathrm{CDCl}_{3}\right): \delta 174.5,171.6,132.1,131.2,130.8,130.5$, $129.8,124.4,70.4,52.2,41.7,41.2,40.7,36.5$ and $18.0 \mathrm{ppm}$; IR (thin film) $v_{\max } 2996,1765,1740$, 1440, 1003, 972, $740 \mathrm{~cm}^{-1}$; MS (70 eV, EI) m/z (\%): 262 (14) [M]+, 231 (33), 203 (35), 91 (100), 77 (20); Anal. Calcd for $\mathrm{C}_{15} \mathrm{H}_{18} \mathrm{O}_{4}$ : C, 68.68; H, 6.92. Found: C, 69.02; H, 7.05.

Tetracycle 5: white solid; $\mathrm{mp} 178-180{ }^{\circ} \mathrm{C}$ (hexane/ethyl acetate); $\mathrm{R}_{\mathrm{f}}=0.35$; hexane/ethyl acetate (1:1); ${ }^{1} \mathrm{H}$ NMR (300 MHz, $\left.\mathrm{CDCl}_{3}\right): \delta 6.08$ (1H, ddd, $J=9.1,3.3,3.3 \mathrm{~Hz}, \mathrm{H} 6$ '), 5.98 (2H, s, H6, H7), $5.87\left(1 \mathrm{H}, \mathrm{ddd}, J=9.2,3.2,3.2 \mathrm{~Hz}, \mathrm{H} 5^{\prime}\right), 4.55(1 \mathrm{H}, \mathrm{dd}, J=7.9,7.3 \mathrm{~Hz}, \mathrm{H} 1), 3.89(1 \mathrm{H}, \mathrm{dd}, J=11.4,8.1$ $\mathrm{Hz}, \mathrm{H} 1), 3.79(1 \mathrm{H}, \mathrm{dd}, J=9.2,5.0 \mathrm{~Hz}, \mathrm{H7a}), 3.73\left(3 \mathrm{H}, \mathrm{s},-\mathrm{CO}_{2} \mathrm{CH}_{3}\right), 3.62-3.68(1 \mathrm{H}, \mathrm{m}, \mathrm{H} 5), 3.34-3.41$ (2H, m, H3a', H4), 3.16-3.29 (1H, m, H7a), 2.42-2.54 (1H, m, H4'), 2.38 (1H, dd, J = 13.7, $3.8 \mathrm{~Hz}$,

H3a), 2.20-2.30 (1H, m, H7') and $1.43\left(3 \mathrm{H}, \mathrm{s},-\mathrm{CH}_{3}\right) \mathrm{ppm} ;{ }^{13} \mathrm{C} \mathrm{NMR}\left(75 \mathrm{MHz} \mathrm{CDCl}_{3}\right): \delta 174.4,171.8$, $171.6,170.8,135.9,131.0,130.7,126.0,70.5,52.5,46.0,43.9,42.3,40.5,39.3,37.9,36.8,30.6$ and 16.1 ppm; IR (KBr disc): $v_{\max } 3032,2984,1838,1769,1724,1062 \mathrm{~cm}^{-1}$; MS (70 eV, EI) m/z (\%): 360 (15) $[M]^{+}, 329$ (100), 301 (75), 299 (75); Anal. Calcd for $\mathrm{C}_{19} \mathrm{H}_{20} \mathrm{O}_{7}$ : C, 63.33; H, 5.59. Found: C, 63.56; H, 5.43.

Tetracycle 6: white solid; $\mathrm{mp} 123-126^{\circ} \mathrm{C}$ (hexane/ethyl acetate); $\mathrm{R}_{\mathrm{f}}=0.17$; hexane/ethyl acetate (1:1); ${ }^{1} \mathrm{H}$ NMR $\left(300 \mathrm{MHz}, \mathrm{CD}_{3} \mathrm{CN}\right): \delta 6.14(1 \mathrm{H}, \mathrm{ddd}, J=10.2,3.0,3.0 \mathrm{~Hz}, \mathrm{H} 7), 6.06$ (1H, ddd [partially obscured], $J=10.2,1.4,1.4 \mathrm{~Hz}, \mathrm{H6}), 6.04$ (1H, ddd [partially obscured], $J=8.8,3.3,3.3 \mathrm{~Hz}, \mathrm{H} 6$ '), 5.90 $\left(1 \mathrm{H}, \mathrm{ddd}, J=9.2,3.2,3.2 \mathrm{~Hz}, \mathrm{H} 5^{\prime}\right), 4.54(1 \mathrm{H}, \mathrm{dd}, J=7.7,7.2 \mathrm{~Hz}, \mathrm{H} 1), 3.85(1 \mathrm{H}, \mathrm{dd}, J=11.4,8.0 \mathrm{~Hz}$, H1), $3.70\left(1 \mathrm{H}, \mathrm{dd}, J=9.4,5.2 \mathrm{~Hz}, \mathrm{H} 7 \mathrm{a}^{\prime}\right), 3.68\left(3 \mathrm{H}, \mathrm{s},-\mathrm{CO}_{2} \mathrm{CH}_{3}\right), 3.43\left(1 \mathrm{H}, \mathrm{dd}, J=9.2,7.6 \mathrm{~Hz}, \mathrm{H} 3 \mathrm{a}^{\prime}\right)$, 3.26-3.34 (1H, m [partially obscured], H5), 3.16-3.27 (1H, m [partially obscured], H7a), 3.06 (1H, d, J $=3.3 \mathrm{~Hz}, \mathrm{H} 4), 2.52(1 \mathrm{H}, \mathrm{dd}, J=13.6,3.4 \mathrm{~Hz}, \mathrm{H} 3 \mathrm{a}), 2.44-2.57(1 \mathrm{H}, \mathrm{m}$ [obscured], H4'), 2.26-2.36 (1H, $\mathrm{m}, \mathrm{H} 7)$ and $1.35\left(3 \mathrm{H}, \mathrm{s},-\mathrm{CH}_{3}\right) \mathrm{ppm} ;{ }^{13} \mathrm{C} \mathrm{NMR}\left(75 \mathrm{MHz} \mathrm{CD}_{3} \mathrm{CN}\right): \delta 175.4,173.8,172.8,172.7,137.0$, $133.1,130.3,127.6,71.5,52.8,47.5,44.7,41.9,41.6,41.3,40.5,37.6,31.1$ and $16.3 \mathrm{ppm}$; IR $(\mathrm{KBr}$ disc): $v_{\max } 3018,2891,1754,1711,1444 \mathrm{~cm}^{-1}$; MS (70 eV, EI) m/z (\%): 360 (4) [M] $]^{+}, 329$ (45), 301 (100), 299 (75), 159 (23); Anal. Calcd for $\mathrm{C}_{19} \mathrm{H}_{20} \mathrm{O}_{7}$ : C, 63.33; H, 5.59. Found: C, 63.49; H, 5.65. 


\section{Intermolecular Diels-Alder-Intermolecular Diels-Alder Sequence: Using 1.0 molar equivalent of}

maleic anhydride. To a stirred solution of tetraenol $1(0.10 \mathrm{mg}, 0.67 \mathrm{mmol})$, and BHT (15.0 mg, 68 $\mu \mathrm{mol})$ in toluene $(2.0 \mathrm{~mL})$ was added maleic anhydride $(65.3 \mathrm{mg}, 0.67 \mathrm{mmol})$ and the resulting solution heated at reflux for $8 \mathrm{~h}$. After removal of the solvent in vacuo, the crude residue was purified by HPLC eluting with hexane/ethyl acetate (5:2) to afford 7 (42.0 mg, $160 \mu \mathrm{mol}, 21 \%), 8$ (37.1 mg, $156 \mu \mathrm{mol}$, $20 \%)$ and 9 (64.8 mg, $180 \mu \mathrm{mol}, 24 \%)$ and starting material 1 (25.1 mg, $171 \mu \mathrm{mol})$ (ratio 7:8:9:1 = $26: 24: 23: 27)$.

Bicyclic adduct 7: colorless oil; $t_{\mathrm{R}}=27.6 \mathrm{~min}$, hexane/ethyl acetate $(5: 2) ; \mathrm{R}_{\mathrm{f}}=0.23$; hexane/ethyl acetate $(2: 1) ;{ }^{1} \mathrm{H}$ NMR (300 MHz, $\left.\mathrm{CDCl}_{3}\right): \delta 5.95(2 \mathrm{H}, \mathrm{m}, \mathrm{H} 3$ ', H4'), $5.90(1 \mathrm{H}, \mathrm{ddd}, J=10.0,4.9,2.1$ Hz, H7), 5.54-5.75 (3H, m, H1', H2', H6), 4.39 (1H, dd, $J=8.6,7.5$ Hz, H1), 4.14 (1H, dd, $J=8.8,3.0$ Hz, H1), 3.42 (1H, dd, J = 9.1, 4.7 Hz, H4), 3.31-3.34 (1H, m [partially obscured], H5), 3.17-3.27 (1H, m, H7a), $3.08(1 \mathrm{H}, \mathrm{dd}, J=5.1,5.1 \mathrm{H} 3 \mathrm{a})$ and $1.71\left(3 \mathrm{H}, \mathrm{d}, J=6.9 \mathrm{~Hz},-\mathrm{CH}_{3}\right) \mathrm{ppm} ;{ }^{13} \mathrm{C} \mathrm{NMR}(75 \mathrm{MHz}$ $\left.\mathrm{CDCl}_{3}\right): \delta 176.3,171.3,132.6,131.4,131.3,129.3,128.7,126.0,71.0,51.9,41.8,38.0,37.6,35.7,18.0$ ppm; IR (thin film): $v_{\max } 2986,1772,1641,1218 \mathrm{~cm}^{-1}$; MS (70 eV, EI) m/z (\%): $262(45)[\mathrm{M}]^{+}, 230$ (100), 202 (78), 105 (12), 93 (20); Anal. Calcd for $\mathrm{C}_{15} \mathrm{H}_{18} \mathrm{O}_{4}$ : C, 68.68; H, 6.92. Found: C, 68.96; H, 7.02 .

Bicyclic adduct 8: colorless oil; $\mathrm{R}_{\mathrm{f}}=13.8$; hexane/ethyl acetate (5:2); ${ }^{1} \mathrm{H}$ NMR $\left(300 \mathrm{MHz}, \mathrm{CDCl}_{3}\right): \delta$ $6.37(1 \mathrm{H}, \mathrm{ddd}, J=15.0,11.0,1.3 \mathrm{~Hz}, \mathrm{H} 3 '), 6.25\left(1 \mathrm{H}, \mathrm{dd}, J=15.0,9.5 \mathrm{~Hz}, \mathrm{H} 2^{\prime}\right), 6.14(1 \mathrm{H}, \mathrm{dd}, J=14.8$, 8.0 Hz, H1'), 5.80-5.95 (3H, m, H4', H5, H6), 4.21 (1H, dd, J = 5.9, 1.2 Hz, H5'), 3.42 (1H, dd, $J=9.2$, $6.1 \mathrm{~Hz}, \mathrm{H} 7 \mathrm{a}), 3.32$ (1H, dd, $J=9.3,7.0 \mathrm{~Hz}, \mathrm{H3a}), 3.00-3.08(1 \mathrm{H}, \mathrm{m}, \mathrm{H} 4 '), 2.45-2.57$ (1H, m, H7'), 1.64 $(1 \mathrm{H}$, br. s, $-\mathrm{OH})$ and $1.46\left(3 \mathrm{H}, \mathrm{d}, J=7.3 \mathrm{~Hz},-\mathrm{CH}_{3}\right) \mathrm{ppm} ;{ }^{13} \mathrm{C} \mathrm{NMR}\left(75 \mathrm{MHz} \mathrm{CDCl}_{3}\right): \delta 171.2,171.0$, $134.4,132.4,132.1,131.8,131.3,130.6,63.2,47.1,45.7,38.8,30.5$ and 16.4 ppm; IR (thin film): $v_{\max }$ 3423, 2953, 1770, 1732, 1436, $1209 \mathrm{~cm}^{-1}$; MS (70 eV, EI) m/z (\%): 248 (18) [M] $]^{+}, 220$ (100), 203 (23), 192 (54), 159 (25); Anal. Calcd for $\mathrm{C}_{14} \mathrm{H}_{16} \mathrm{O}_{4}$ : C, 67.73; H, 6.50. Found: C, 67.84; H, 6.66. 
Double cycloadduct 9: white solid, $\mathrm{mp} 178-181{ }^{\circ} \mathrm{C}$ (hexane/THF); $\mathrm{R}_{\mathrm{f}}=0.24$; hexane/ethyl acetate (1:1); ${ }^{1} \mathrm{H}$ NMR (300 MHz, $\left.\mathrm{CDCl}_{3}\right): \delta 5.86-5.89(2 \mathrm{H}, \mathrm{m}, \mathrm{H} 5$ ', H6'), $5.80(1 \mathrm{H}, \mathrm{ddd}, J=10.0,2.6,2.6 \mathrm{~Hz}$, H7), 5.68 (1H ddd, $J=9.9,2.3,2.3 \mathrm{~Hz}, \mathrm{H6}), 4.59$ (1H, dd, $J=9.2,8.3 \mathrm{~Hz}, \mathrm{H1}), 4.11(1 \mathrm{H}, \mathrm{dd}, J=8.3$, $8.5 \mathrm{~Hz}, \mathrm{H1}), 3.99$ (1H, dd, $J=9.4,5.7 \mathrm{~Hz}, \mathrm{H} 7 \mathrm{a}), 3.74(1 \mathrm{H}, \mathrm{br} . \mathrm{dd}, J=3.9,3.9 \mathrm{~Hz}, \mathrm{H} 4), 3.67$ (3H, s, $\left.-\mathrm{CO}_{2} \mathrm{CH}_{3}\right), 3.39(1 \mathrm{H}, \mathrm{dd}, J=9.4,7.2 \mathrm{~Hz}, \mathrm{H} 3 \mathrm{a}), 3.25-3.34(1 \mathrm{H}, \mathrm{m}$ [partially obscured], H7a), $3.20(1 \mathrm{H}$, dd, $J=11.5,4.7 \mathrm{~Hz}, \mathrm{H} 5), 2.44-2.56\left(2 \mathrm{H}, \mathrm{m}, \mathrm{H} 4{ }^{\prime}, \mathrm{H} 77^{\prime}\right)$ and $1.46\left(3 \mathrm{H}, \mathrm{d}, J=7.3 \mathrm{~Hz}, \mathrm{CH}_{3}\right) \mathrm{ppm} ;{ }^{13} \mathrm{C} \mathrm{NMR}$ (75 $\left.\mathrm{MHz} \mathrm{CDCl}_{3}\right): \delta 177.3,172.2,171.4,170.9,135.8,131.5$ [two coincident resonances], 128.3, 71.5, 52.1, 46.1 [two coincident resonances], 40.9, 40.2, 38.0 [two coincident resonances], 33.6, 30.7 and 16.1 ppm; IR (thin film): $v_{\max } 3033,2927,1770,1726,1222 \mathrm{~cm}^{-1}$; MS (70 eV, EI) $\mathrm{m} / z(\%): 360$ (100) $[M]^{+}$, 329 (69), 301 (23), 299 (65), 159 (76); Anal. Calcd for $\mathrm{C}_{19} \mathrm{H}_{20} \mathrm{O}_{7}$ : C, 63.33; H, 5.59. Found: C, 63.72; H, 5.74 .

\section{Intermolecular Diels-Alder-Intermolecular Diels-Alder Sequence: Using 2.0 molar equivalents} of maleic anhydride. To a stirred solution of tetraenol $1(0.12 \mathrm{mg}, 0.80 \mathrm{mmol})$, and BHT (21.0 $\mathrm{mg}, 95$ $\mu \mathrm{mol})$ in toluene $(2.0 \mathrm{~mL})$ was added maleic anhydride $(0.16 \mathrm{~g}, 1.68 \mathrm{mmol})$ and the resulting solution heated at reflux for $8 \mathrm{~h}$. After removal of the solvent in vacuo, the crude residue was purified over silica gel $(20 \mathrm{~g})$ eluting with hexane/ethyl acetate (1:1) to afford, exclusively, tetracylic adduct $9(0.28 \mathrm{~g}, 0.77$ mmol, 96\%). 


\section{References}

(1) Perrin, D. D.; Armarego, W. L. F. Purification of Laboratory Chemicals; Pergamon: Oxford, 1988.

(2) Tetraenol 1 has been prepared by a five step synthesis starting from available sorbaldehyde: Ley, S. V.; Smith, S. C.; Woodward, P. R. Tetrahedron 1992, 48, 1145-1174. We prepare this compound on large scale in three steps from crotonaldehyde, through self condensation to $2 E, 4 E, 6 E$-octatrienal (D'Amico, $\mathrm{K}$. L.; Manos, C.; Christensen, R. L. J. Am. Chem. Soc. 1980, 102, 1777-1782), Wittig reaction and DIBAL reduction. 
$300 \mathrm{MHz}{ }^{1} \mathrm{H}$ NMR spectrum in $\mathrm{d}_{6}-\mathrm{DMSO}$
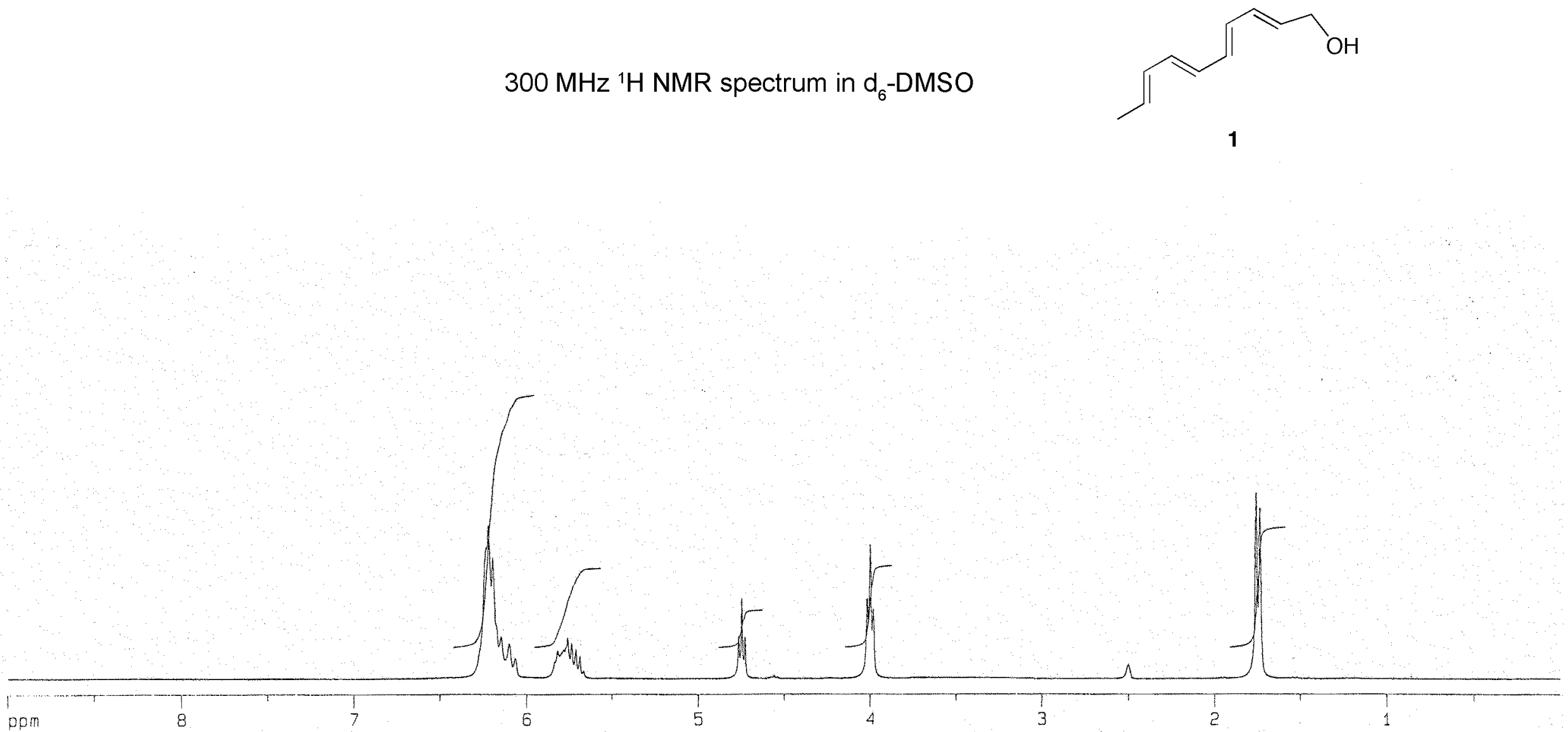
$75 \mathrm{MHz}{ }^{13} \mathrm{C}$ NMR spectrum in $\mathrm{d}_{6}$-DMSO
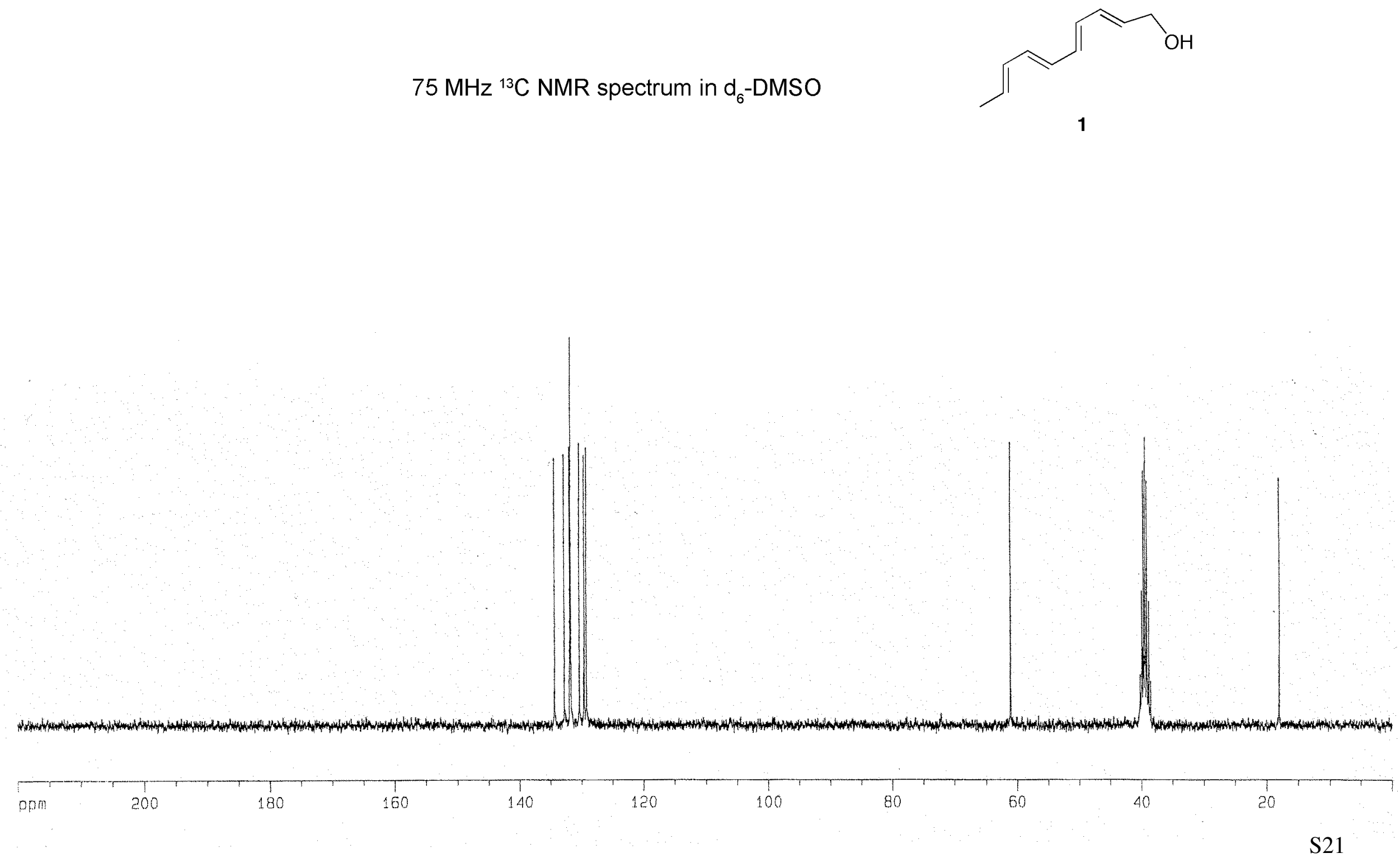
$300 \mathrm{MHz}{ }^{1} \mathrm{H}$ NMR spectrum in $\mathrm{CDCl}_{3}$
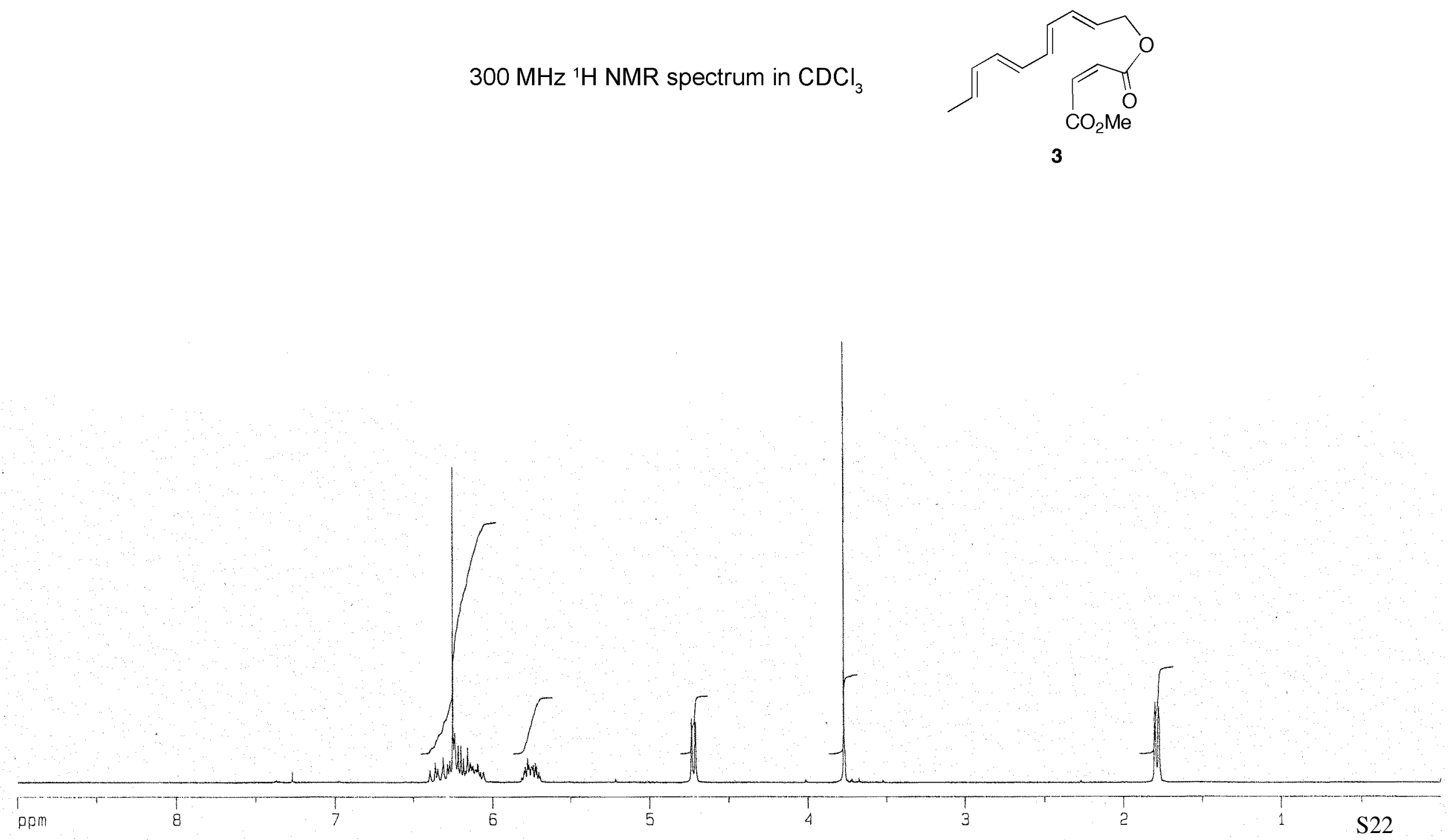

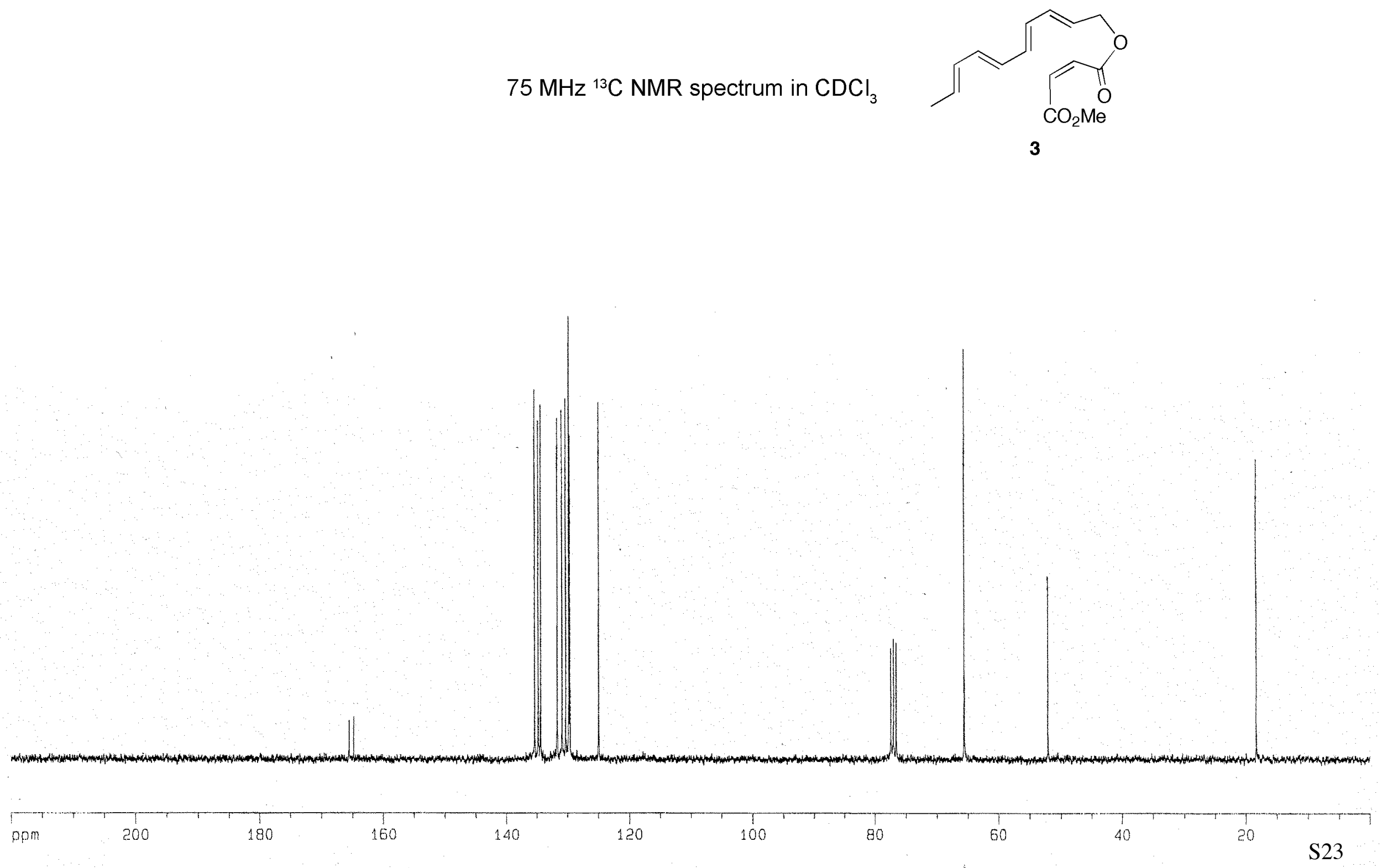
$300 \mathrm{MHz}{ }^{1} \mathrm{H}$ NMR spectrum in $\mathrm{CDCl}_{3}$
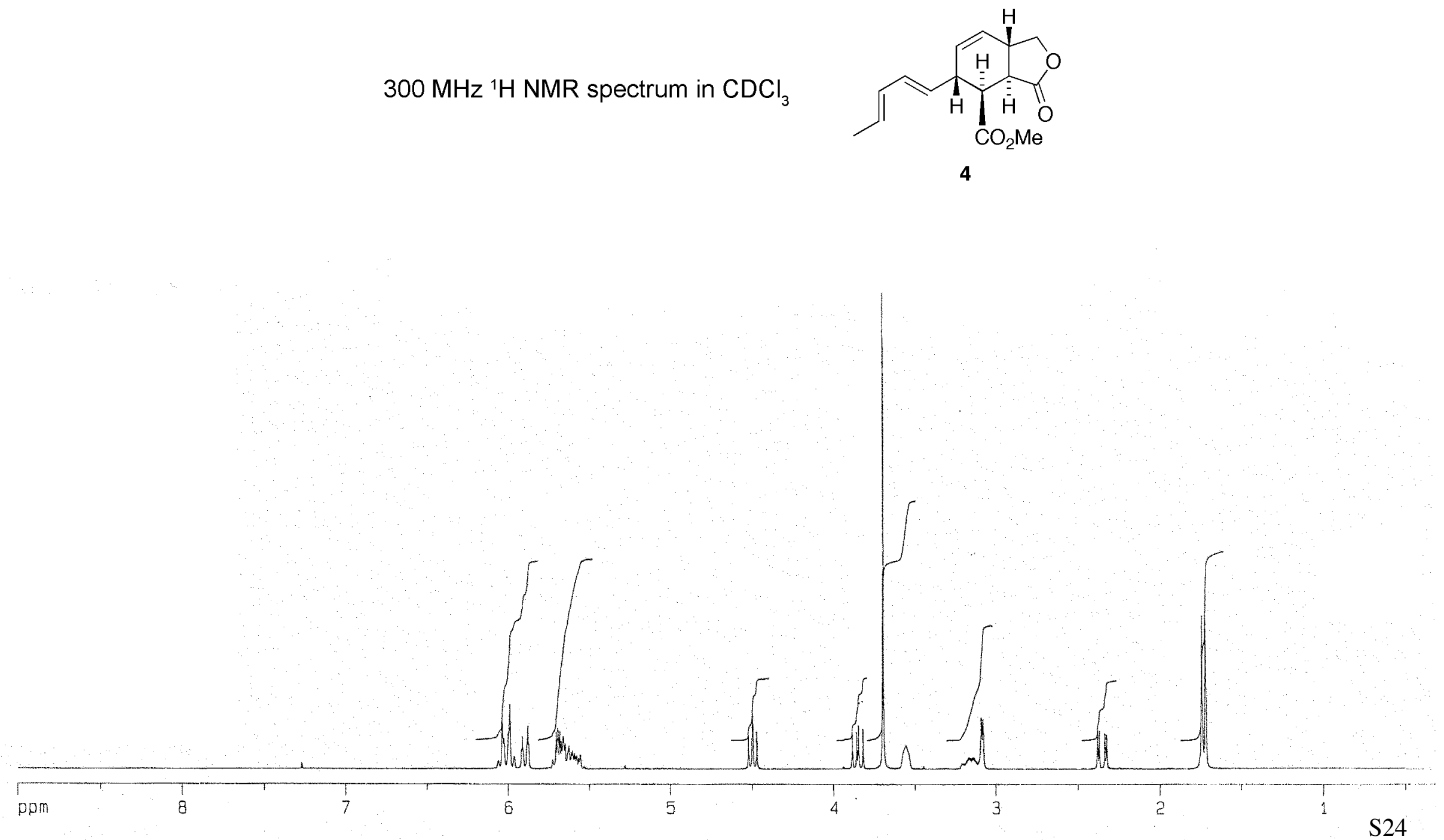

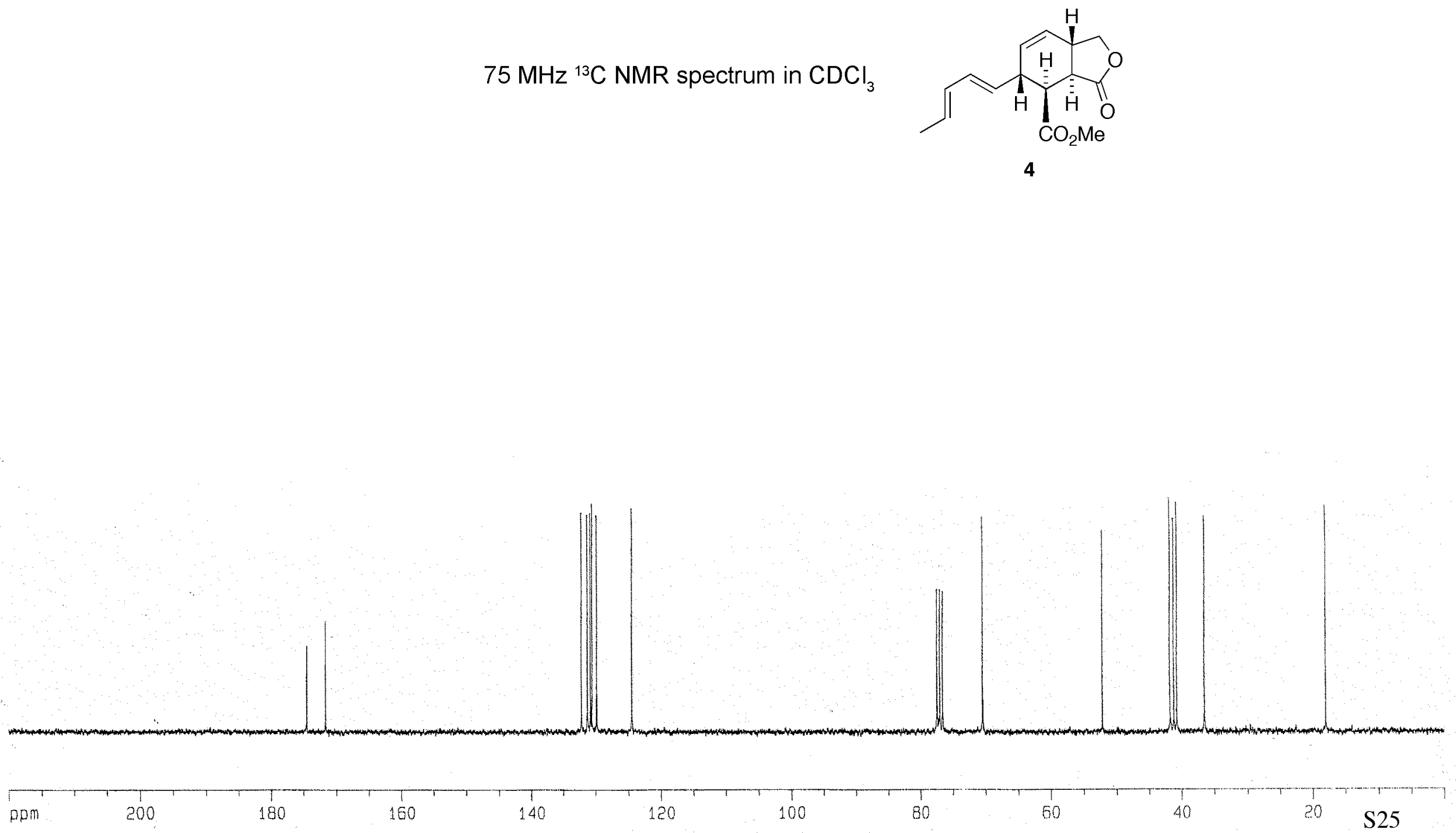
$300 \mathrm{MHz}{ }^{1} \mathrm{H}$ NMR spectrum in $\mathrm{CDCl}_{3}$
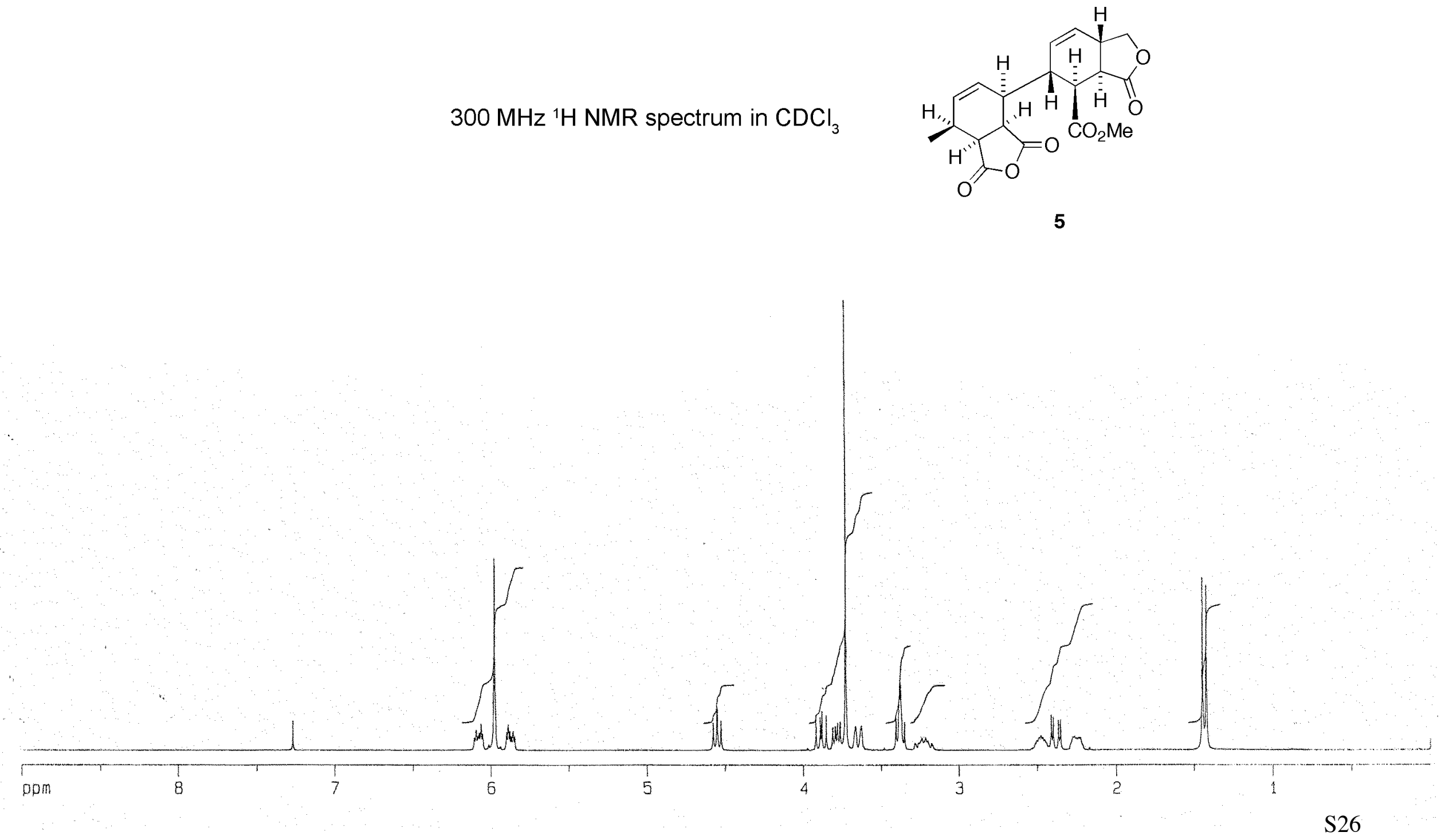
$75 \mathrm{MHz}{ }^{13} \mathrm{C}$ NMR spectrum in $\mathrm{CDCl}_{3}$

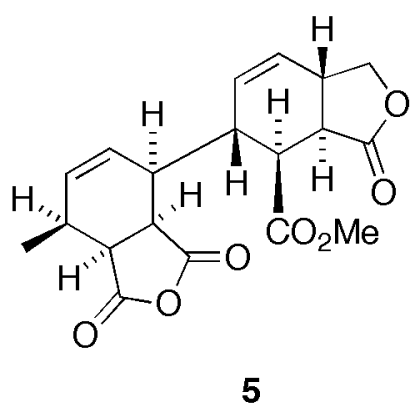

||||

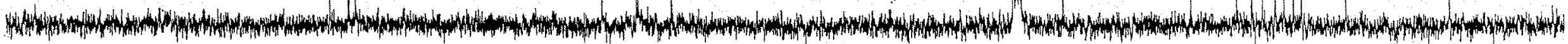



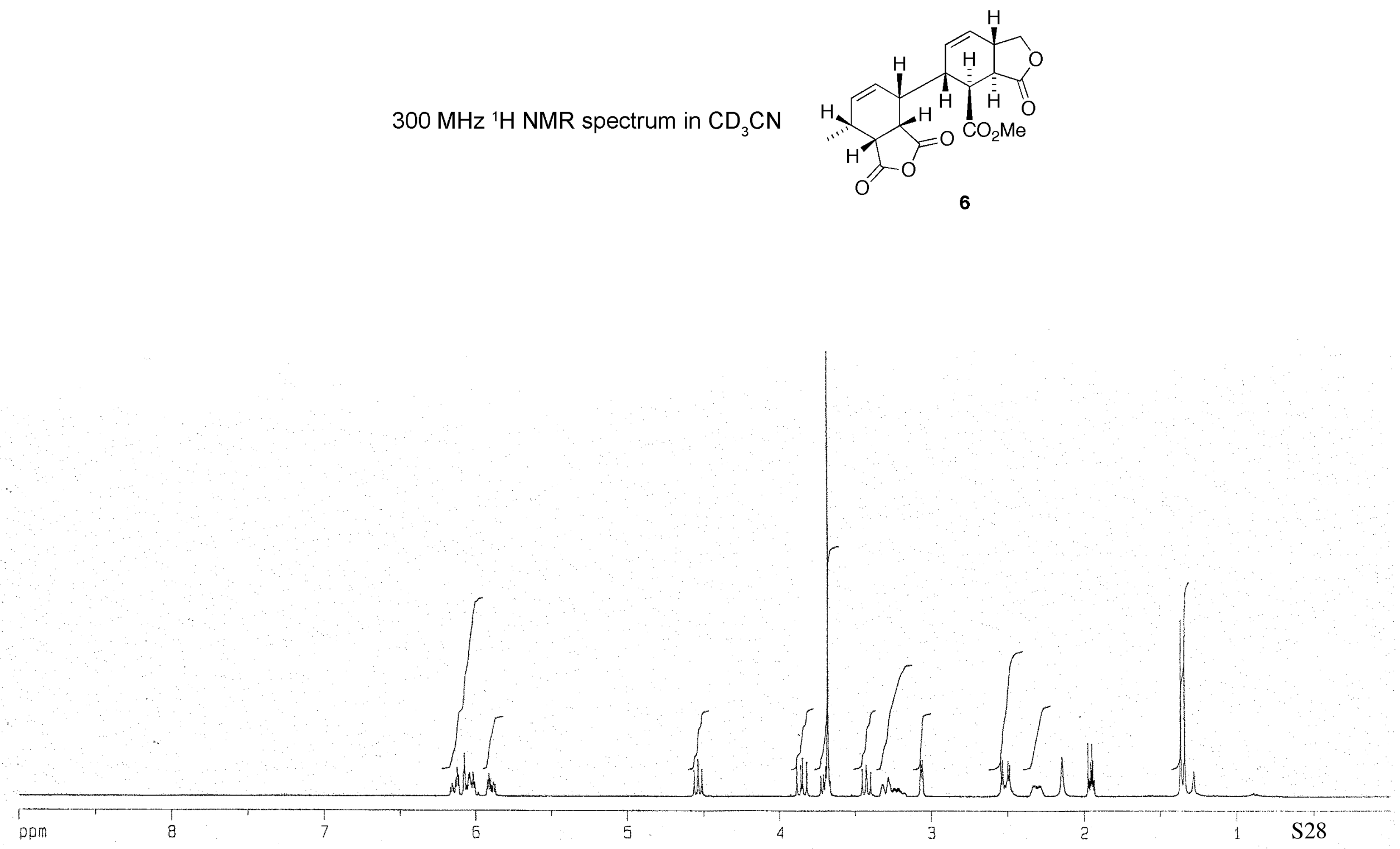
$75 \mathrm{MHz}{ }^{13} \mathrm{C}$ NMR spectrum in $\mathrm{CD}_{3} \mathrm{CN}$

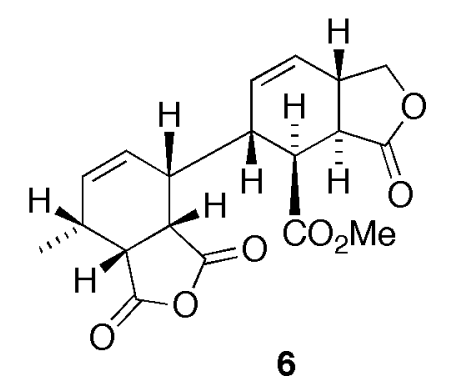



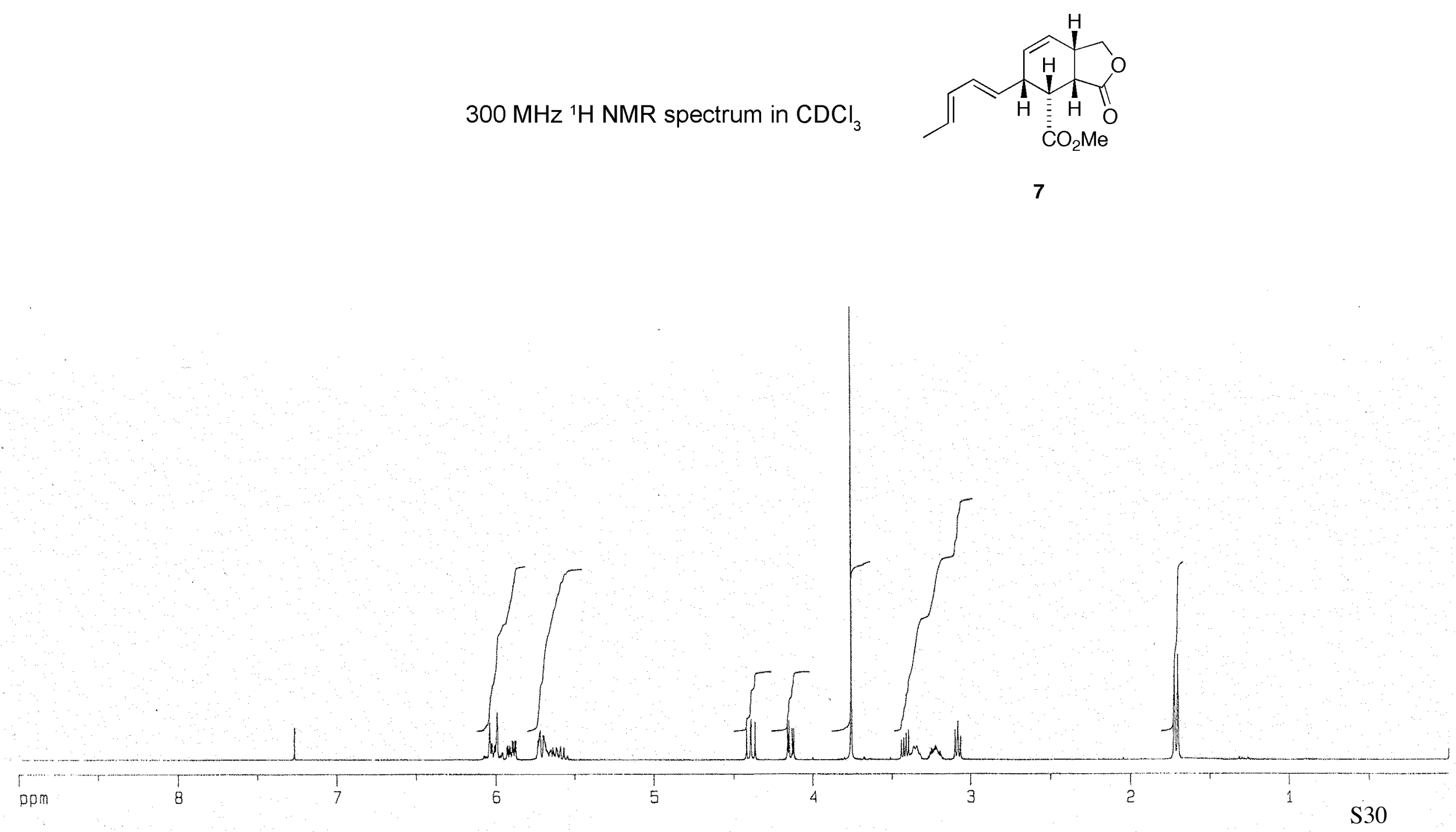
$75 \mathrm{MHz}{ }^{13} \mathrm{C}$ NMR spectrum in $\mathrm{CDCl}_{3}$
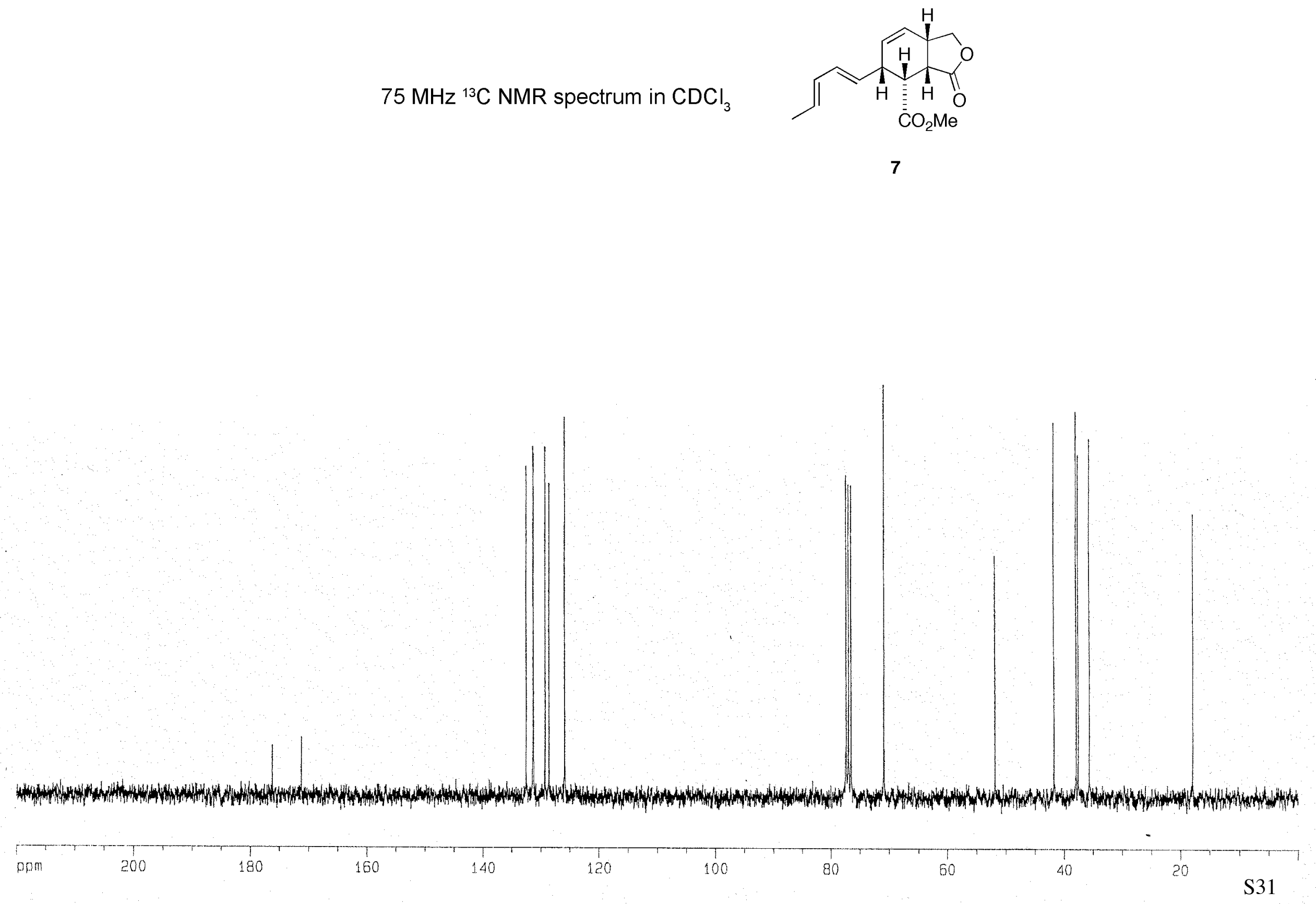

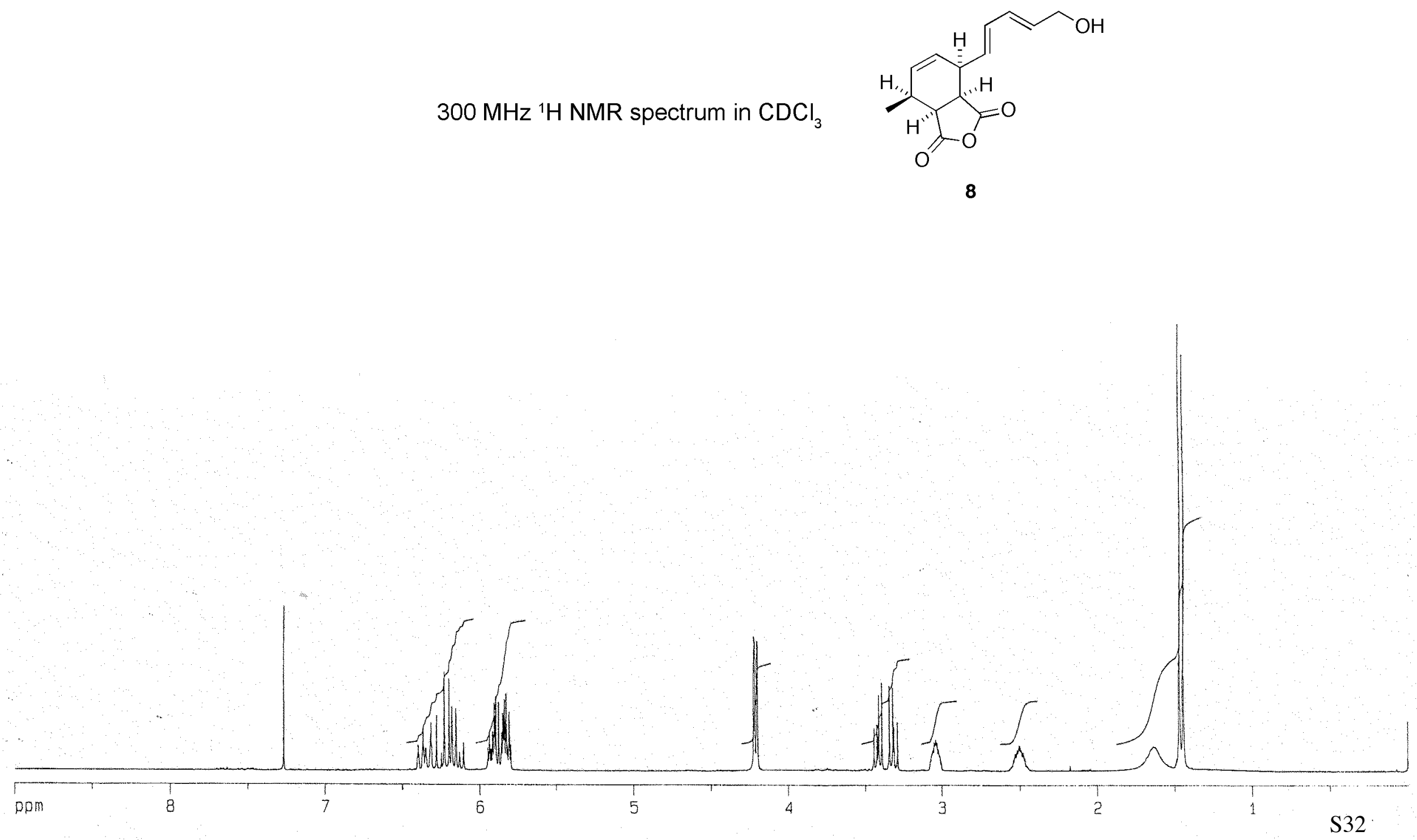
$75 \mathrm{MHz}{ }^{13} \mathrm{C}$ NMR spectrum in $\mathrm{CDCl}_{3}$
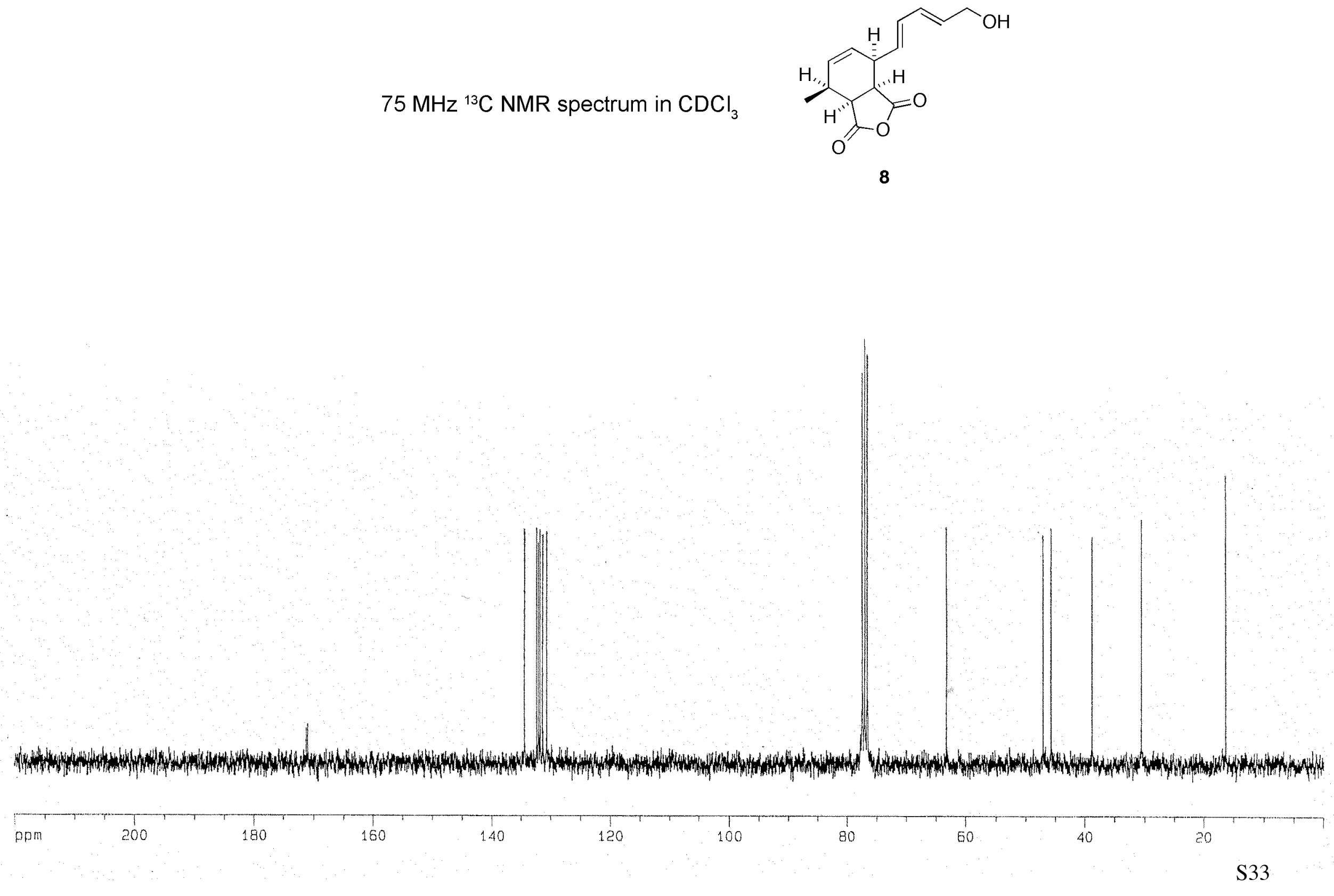


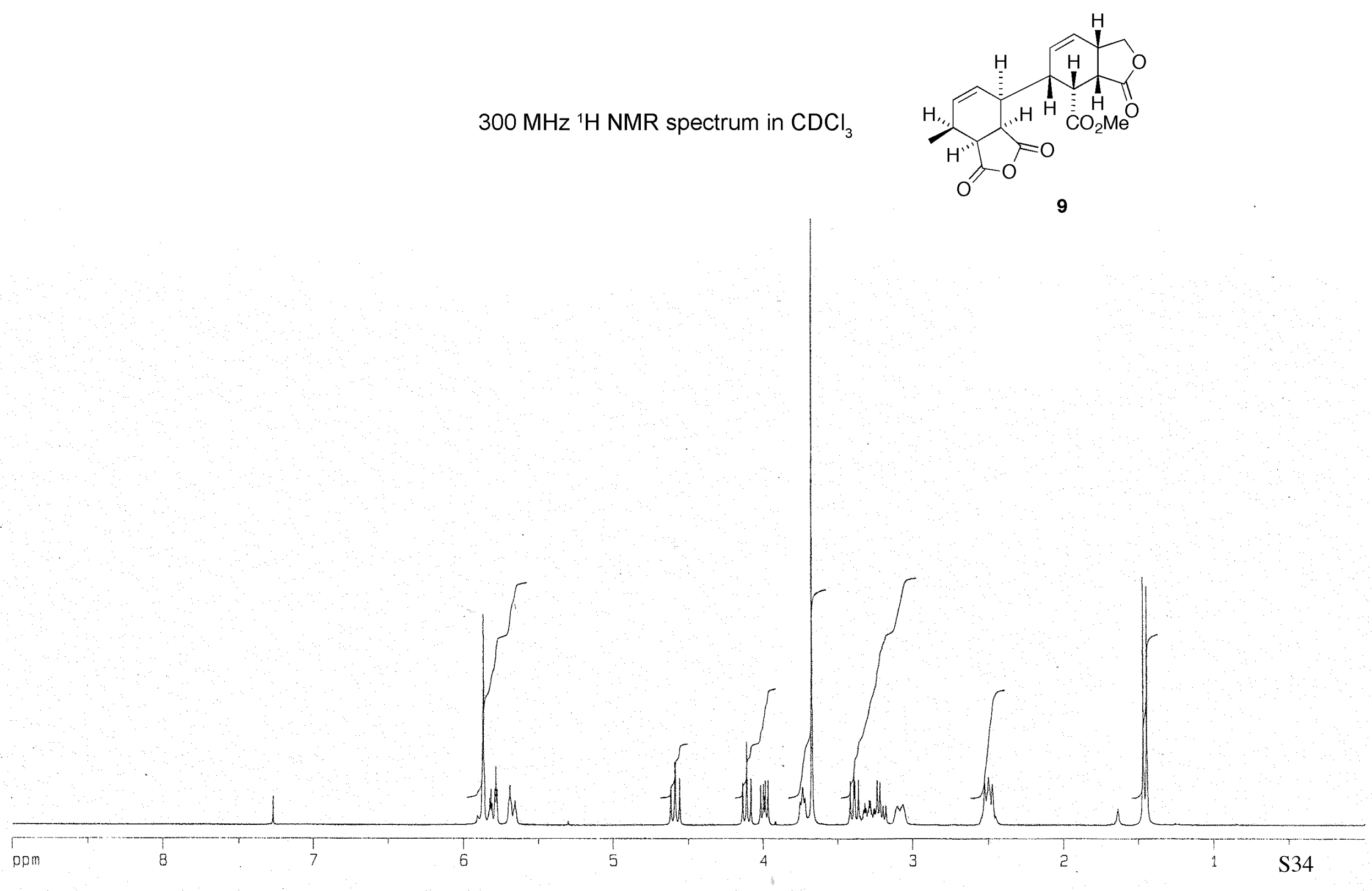


$75 \mathrm{MHz}{ }^{13} \mathrm{C}$ NMR spectrum in $\mathrm{CDCl}_{3}$
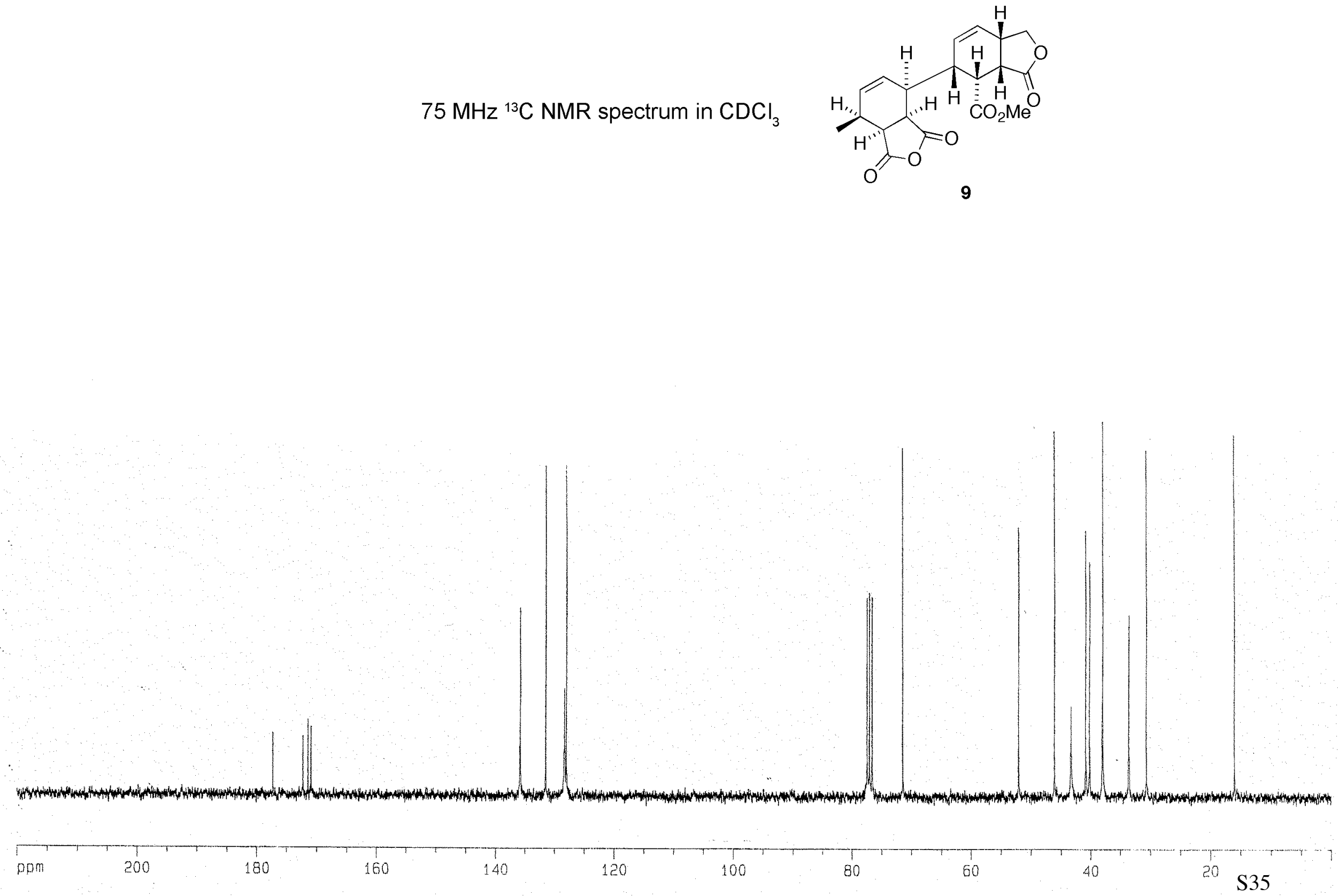